\title{
Crystal-field model study of the xenon hexafluoride molecule. I. Energy levels and molecular geometry
}

\author{
Sylvester Y. Wang and Lawrence L. Lohr, Jr. \\ Department of Chemistry, The University of Michigan, Ann Arbor, Michigan 48104
}

(Received 30 April 1973)

\begin{abstract}
A crystal field approach is used to examine theoretically the energy levels and wavefunctions for a gaseous xenon hexafluoride molecule. The electronic configurations used are $5 s^{2}, 5 s 5 p$, and $5 p^{2}$ of hexavalent xenon. Four electronic parameters are used to explore the variation in energy with the amount of distortion from $0_{h}$ symmetry. These are the energy difference between the xenon $5 s$ and $5 p$ orbitals, the spin-orbit coupling constant for xenon $5 p$, and two Slater-Condon electron repulsion parameters. Pure bending vibrational modes of $t_{1 u}, t_{2 g}$, and $t_{2 u}$ symmetries are examined. The mixing of xenon $5 s$ and $5 p$ orbitals by the $t_{i u}$ vibration is particularly important, leading to a possible pseudo-Jahn-Teller distortion from $0_{h}$ symmetry. Calculations of the vibrational force constants and frequencies are made for the bending modes, and the results are compared with observed values for other hexafluorides and related complexes. Calculations made on the coupling between $t_{1 u}$ and $t_{2 g}$ modes indicate an enhanced stabilization of the $C_{3 \nu}$ structure. These studies lend support to the interpretation of the observed anomalous properties of the xenon hexafluoride molecule in terms of a nonrigid octahedral structure but not to interpretations requiring thermally populated spin-triplet excited states.
\end{abstract}

\section{INTRODUCTION}

Of the known noble-gas compounds, perhaps none has proved as elusive in its characterization as $\mathrm{XeF}_{6}$. The early experimental and theoretical investigations were reported at the first conference devoted to noble-gas compounds, ${ }^{1}$ while later research on $\mathrm{XeF}_{6}$ and related compounds has been reviewed by Malm et al. , ${ }^{2}$ Claassen, ${ }^{3}$ and Hyman. ${ }^{4}$ An important early theoretical paper on the xenon fluorides is that of Coulson. ${ }^{5}$

We begin by noting some of the properties of $\mathrm{XeF}_{6}$ which seem particularly unusual when compared to those of other ${ }^{6}$ hexafluorides:

(1) $\mathrm{XeF}_{6}$ is a white solid at room temperature, but acquires a yellowish color somewhat below its melting point of $49.5^{\circ} \mathrm{C}$, while careful heat capacity measurements indicated no evidence of any phase change near the melting point. ${ }^{7}$

(2) The liquid range for $\mathrm{XeF}_{6}\left(26^{\circ} \mathrm{C}\right.$ at $\left.1 \mathrm{~atm}\right)$ is longer and the vapor pressure is lower than for any other known hexafluoride. Its liquid has high entropy of vaporization ( 32.7 e.u.) and a high and rapidly rising heat capacity. 7

(3) The solubility in anhydrous $\mathrm{HF}$ for $\mathrm{XeF}_{6}$ is exceptionally large for a hexafluoride. It is also substantially ionized in contradiction to all the other hexafluorides investigated. ${ }^{8}$

(4) Analysis of the ir and Raman spectra of $\mathrm{XeF}_{6}$ based on the assumption that the gaseous molecule has $O_{h}$ symmetry, as have all the other hexafluorides, has been unsuccessful, indicating that $\mathrm{XeF}_{6}$ is different from the other hexafluorides. ${ }^{9-14}$

(5) Using $\mathrm{He}-\mathrm{Ne}$ laser light source with an intense red line at $6328 \mathrm{~A}$, a virtual coincidence is found between the observed ir bands and Raman bands for $\mathrm{XeF}_{B}$, which would be forbidden in a molecule with a center of symmetry. ${ }^{13}$

(6) Electron diffraction studies on gaseous $\mathrm{XeF}_{6}$ reveal large amplitudes of displacement away from $O_{h}$ symmetry, strongly suggesting that the molecule does not have an $O_{h}$ equilibrium geometry. However it has proven difficult to find an unequivocal arrangement that satisfies the diffraction data. ${ }^{15-18}$

(7) The ions isovalent with $\mathrm{XeF}_{6}$, such as $\mathrm{TeCl}_{6}^{-2}$, $\mathrm{TeBr}_{6}^{-2}$, and $\mathrm{SbBr}_{\mathrm{B}}^{-3}$, are known to possess definite $O_{h}$ symmetry in the solid phase. ${ }^{19-22}$

At least two schools of thought exist today which attempt to explain these abnormalities. One is that represented by Goodman, ${ }^{23}$ who concentrates on interpreting the ir and visible spectra ${ }^{14}$ in terms of an excited state Jahn-Teller distortion. It is proposed in this view that there are three electronic isomers for $\mathrm{XeF}_{B}$, namely a spin-singlet ground state of $O_{h}$ symmetry and spintriplet excited states of $D_{3 d}$ (oblate) and $D_{3 d}$ (prolate) symmetries, with the latter two states being thermally accessible at room temperature. However, magnetic experiments have not yet been able to confirm these lowlying triplet states. ${ }^{24,25}$ None of the photoelectron spectroscopy work by Brundle, Jones, and Basch, ${ }^{26}$ the photoionization mass spectroscopy work by Berkowitz, et al. ${ }^{27}$ and the threshold electron-impact work by Begun and Compton ${ }^{28}$ have been able to give support to this interpretation. The other school is the idea of a pseudoJahn-Teller distortion of the ground state, an idea derived from Gillispie's model ${ }^{29}$ and developed by Gavin and Bartell. 17,30,31 It is argued that if we start with the Gillespie model, we can consider two modifications of $O_{h}$ as being most likely. The lone pair electrons may be mainly concentrated in the belt or in the face of an octahedron corresponding to a $C_{2 v}$ or $C_{3 v}$ symmetry, respectively. In either case, the lone pair repulsion will distort the octahedral bond angles. To account for the negligible dipole observed in a molecular beam experiment, ${ }^{25}$ the molecule is thought to have rapid inverting structures (pseudorotation), with inversion of the electron pair and wide oscillations of the fluorine atoms. However, this predicts a far ir or microwave absorption which is yet to be found. ${ }^{30}$

We have treated the problem of $\mathrm{XeF}_{6}$ in the spirit of 
Gavin and Bartell's interpretation, but using a simple crystal field model as a basis for quantitative calculations. In this model we consider $\mathrm{XeF}_{6}$ as being formed by six fluoride ligands surrounding a $\mathrm{Xe}(+6)$ ion with two electrons outside a $\mathrm{Te}(+6)$-like core, these two electrons corresponding to the lone pair. We have then used the results of our calculations to obtain a semiquantitative interpretation of the anomalous properties of the $\mathrm{XeF}_{6}$ molecule.

\section{CRYSTAL FIELD MODEL}

In our crystal field approach to the study of $\mathrm{XeF}_{6}$, we approximate the electronic structure of the molecule as that of a central ion $\mathrm{Xe}(+6)$ subjected to the electric field created by the six negative fluoride ions surrounding the $\mathrm{Xe}(+6)$ ion. We therefore first examine the states arising for the $\mathrm{Xe}(+6)$ ion. These are the states for two electrons outside a $\mathrm{Te}(+6)$-like closed shell core and are associated with the configurations $5 s^{2}, 5 s 5 p$, and $5 p^{2}$. Configurations involving $5 d$ and higher orbitals are not considered. The terms arising from the configurations involving only $5 s$ and/or $5 p$ are ${ }^{1} S$ from $5 s^{2},{ }^{3} p^{0}$, and ${ }^{1} P^{0}$ from $5 s 5 p$, and ${ }^{3} P,{ }^{1} D$, and ${ }^{1} S$ from $5 p^{2}$, giving a total of 28 independent states. It is well-known that the atomic states in the absence of spin-orbit coupling can be classified according to either $\left(E, L, S, M_{L}, M_{S}\right)$ or $\left(E, L, S, J, M_{J}\right)$, since all $J$ levels of a given $L, S$ term are degenerate. In these states designation $E$ is the energy, $L, S$, and $J$ the orbital, spin, and total angular momentum quantum numbers, with $M_{L}, M_{\mathrm{S}}$ and $M_{J}$ being their associated magnetic quantum numbers.

Wavefunctions for the 28 states in either basis can be used to calculate matrix elements of the nonrelativistic Hamiltonian

$$
H_{0}=\sum_{i} f_{i}+\sum_{i>j} g_{i j}
$$

where $f_{i}=-(1 / 2) \nabla_{i}^{2}-Z / r_{i}$, a one-electron operator containing the kinetic energy of the $i$ th electron and the attraction to the nucleus of atomic number $Z$ (in this paper all energies are in atomic units unless otherwise stated) and $g_{i j}$ is the two-electron electron-repulsion operator $r_{i j}^{-1}$. Using conventional notation ${ }^{32,33}$ for the Slater-Condon parameters $F_{k}$ and denoting the contribution from inner closed shells as $I_{0}$, with $I(n l)$ being the expectation value of $f_{i}[\mathrm{Eq} .(1)]$ for the orbital $|n l\rangle$, we readily obtain the following term energies:

$$
\begin{gathered}
5 s^{2}: E\left({ }^{1} S\right)=I_{0}+2 I(5 s)+F_{0}(5 s, 5 s) \\
5 s 5 p: E\left({ }^{3} P^{0}\right)=I_{0}+I(5 s)+I(5 p)+F_{0}(5 s, 5 p) \\
-F_{1}(5 s, 5 p) \\
E\left({ }^{1} P^{0}\right)=I_{0}+I(5 s)+I(5 p)+F_{0}(5 s, 5 p) \\
+F_{1}(5 s, 5 p) ; \\
5 p^{2}: E\left({ }^{3} P\right)=I_{0}+2 I(5 p)+F_{0}(5 p, 5 p)-5 F_{2}(5 p, 5 p) \\
E\left({ }^{1} D\right)=I_{0}+2 I(5 p)+F_{0}(5 p, 5 p)+F_{2}(5 p, 5 p) \\
E\left({ }^{1} S\right)=I_{0}+2 I(5 p)+F_{0}(5 p, 5 p)+10 F_{2}(5 p, 5 p) .
\end{gathered}
$$

There is in addition a nonvanishing off-diagonal matrix element of $H_{0}$ between the two ${ }^{1} S$ terms which can be described as configuration interaction:

$$
\left\langle{ }^{1} S\left(5 s^{2}\right)\left|H_{0}\right|{ }^{1} S\left(5 p^{2}\right)\right\rangle=-\sqrt{3} F_{1}(5 s, 5 p) .
$$

At times it is more convenient to use the average energy of the multiplets, as when we compare theoretical calculations with experimental energy levels. By average we mean a weighted mean in which each multiplet in Russell-Saunders coupling, is given a weight $(2 L+1)$ : $\times(2 S+1)$, equal to the number of separate wavefunctions comprising this multiplet. From this definition, it is simple to show that the average energy of a configuration is given by $^{33}$

$$
E_{\mathrm{Av}}=\sum I_{i}(n, l)+\sum_{i>f} E_{i j}
$$

The interaction energies $E_{i f}$ are

$$
\begin{aligned}
& 5 s^{2}: F_{0}(5 s, 5 s), \\
& 5 s 5 p: F_{0}(5 s, 5 p)-\frac{1}{2} F_{1}(5 s, 5 p), \\
& 5 p^{2}: F_{0}(5 p, 5 p)-2 F_{2}(5 p, 5 p) .
\end{aligned}
$$

From these, we have

$$
\begin{aligned}
& 5 s^{2}: E\left({ }^{1} S\right)=E_{\mathrm{Av}}\left(5 s^{2}\right) \\
& 5 s 5 p: E\left({ }^{3} P^{0}\right)=E_{\mathrm{Av}}(5 s, 5 p)-\frac{1}{2} F_{1}(5 s, 5 p) \\
& E\left({ }^{1} P^{0}\right)=E_{\mathrm{Av}}(5 s, 5 p)+\frac{3}{2} F_{1}(5 s, 5 p) \\
& 5 p^{2}: E\left({ }^{3} P\right)=E_{\mathrm{Av}}\left(5 p^{2}\right)-3 F_{2}(5 p, 5 p) \\
& E\left({ }^{1} D\right)=E_{\mathrm{Av}}\left(5 p^{2}\right)+3 F_{2}(5 p, 5 p) \\
& E\left({ }^{1} S\right)=E_{\mathrm{Av}}\left(5 p^{2}\right)+12 F_{2}(5 p, 5 p) .
\end{aligned}
$$

We have thus far used the Hamiltonian in the form of Eq. (1); however, in a more accurate treatment of the problem, especially for heavy atoms, there is a spinorbit interaction due to the interaction of the relativistic electric dipole moment ${ }^{34}$ of the electron with the electric field within the atom, thus producing the fine structure of the multiplets. In the present treatment, we consider it as a perturbation to Eq. (1). This interaction, in the case of a single electron in an atom can be represented ${ }^{35}$ by

$$
H_{\mathrm{so}}=\frac{\alpha^{2}}{2} \frac{1}{r} \frac{d V}{d r} 1 \cdot \mathbf{s}=\xi(r) 1 \cdot \mathrm{s},
$$

where $\alpha$ is the fine structure constant and $V(r)$ is the electrostatic potential energy due to the central field. The energy $V(r)$ is equal to $-Z / r$ for a nucleus of charge $Z$ when there are no other electrons present. Therefore, $d V / d r$ is positive everywhere, giving a positive sign for the coupling constant $\xi(r)$. For a system of $n$ electrons, a good approximation for the spin-orbit interaction is

$$
H_{\mathrm{so}}=\sum_{i}^{n} \xi\left(r_{i}\right) 1_{i} \cdot \mathbf{s}_{i} .
$$

This we must add to the Hamiltonian in Eq. (1). The selection rules for $H_{\text {so }}$ in a Russell-Saunders basis $\left(E, S, L, J, M_{J}\right)$ are

$$
\begin{aligned}
& \Delta J=0, \\
& \Delta M_{J}=0, \\
& \Delta S=0, \pm 1
\end{aligned}
$$


TABLE I. Nonzero matrix elements of spin-orbit coupling.

\begin{tabular}{lll}
\hline \hline$|i\rangle$ & $|j\rangle$ & \multicolumn{1}{|c}{$\left\langle i\left|H_{s 0}\right| j\right\rangle^{\mathrm{a}}$} \\
\hline${ }^{3} P_{2}^{0}(5 s 5 p)$ & ${ }^{3} P_{2}^{0}(5 s 5 p)$ & $1 / 2$ \\
${ }^{3} P_{1}^{0}(5 s 5 p)$ & ${ }^{3} P_{1}^{0}(5 s 5 p)$ & $-1 / 2$ \\
${ }^{3} P_{0}^{0}(5 s 5 p)$ & ${ }^{3} P_{0}^{0}(5 s 5 p)$ & -1 \\
${ }^{3} P_{2}\left(5 p^{2}\right)$ & ${ }^{3} P_{2}\left(5 p^{2}\right)$ & $1 / 2$ \\
${ }^{3} P_{1}\left(5 p^{2}\right)$ & ${ }^{3} P_{1}\left(5 p^{2}\right)$ & $-1 / 2$ \\
${ }^{3} P_{0}\left(5 p^{2}\right)$ & ${ }^{3} P_{0}\left(5 p^{2}\right)$ & -1 \\
${ }^{3} P_{1}^{0}(5 s 5 p)$ & ${ }^{1} P_{1}^{0}(5 s 5 p)$ & $1 / \sqrt{2}$ \\
${ }^{1} D_{2}\left(5 p^{2}\right)$ & ${ }^{3} P_{2}(5 s 5 p)$ & $1 / \sqrt{2}$ \\
${ }^{3} P_{0}\left(5 p^{2}\right)$ & ${ }^{1} S_{0}\left(5 p^{2}\right)$ & $-\sqrt{2}$ \\
\hline \hline
\end{tabular}

${ }^{\text {an }}$ unit of $\zeta 5 p$.

$$
\begin{aligned}
& \Delta L=0, \pm 1, \text { and } \\
& \Delta \text { parity }=0 .
\end{aligned}
$$

Next, we evaluate the matrix elements of the spinorbit interaction. The most direct effect of spin-orbit interaction is to split a given term into several levels of different $J$. It can be readily shown that

$$
\begin{aligned}
\left\langle{ }^{2 S+1} L_{J M}\left|H_{\text {so }}\right|{ }^{2 S+1} L_{J M}\right\rangle & \\
= & =\frac{1}{2} \lambda[J(J+1)-L(L+1)-S(S+1)],
\end{aligned}
$$

where $\lambda$ is a constant for a given particular term. The difference in energy between the level $J$ and level $J-1$ is $\lambda J$. This result is the Lande interval rule, which states that the separation of two adjacent $J$ states belong to the same $L, S$ is proportional to the larger value of $J$. From Eqs. (8) and (10), we have the sum rule

$$
\sum_{i} \zeta_{n_{i} l_{i}} m_{i_{i}} m_{s_{i}}=\lambda M_{L} M_{S}
$$

where we have defined

$$
\zeta_{n l}=\int_{0}^{\infty} R_{n l}^{2} \xi(r) r^{2} d r .
$$

The spin-orbit coupling constant $\zeta_{n l}$ is positive. The diagonal elements of spin-orbit matrix for the $\mathrm{Xe}(+6)$ are readily obtained for the $\left(E, L, S, J, M_{J}\right)$ basis, and are given in Table I.

In the scheme of basis functions we are using, there are mixing of states due to spin-orbit coupling. These must be obtained using Eq. (8), as the form $\mathrm{L} \cdot \mathrm{S}$ in $\mathrm{Eq}$. (10) does not hold for off-diagonal elements. The resulting nonzero matrix elements are also given in Table I, while Fig. 1 indicates qualitatively the effects of electron repulsion and spin-orbit coupling for the free ion.

We now consider the effect of the crystal field ${ }^{36}$ of the six fluoride ions on the states of $\mathrm{Xe}(+6)$. First consider a point charge $(Q)$ ligand at $\mathbf{R}$, then the potential energy of an electron is

$$
V(\mathbf{r})=-Q /|\mathbf{R}-\mathbf{r}|
$$

where $r$ is the position of the electron around the nucleus (Fig. 2). We now use the expansion

$$
\frac{1}{|\mathbf{R}-\mathbf{r}|}=-\sum_{l} \frac{r_{<}^{l}}{r_{>}^{l+1}} P_{l}(\cos \omega) \text {, }
$$

where $\omega$ is the angle between $\mathbf{r}$ and $\mathbf{R}$ and $r_{<}$is the lesser of $r$ and $R$, with $r$, the greater. Let us assume, as is customary in crystal field treatments, that

$$
\begin{aligned}
& r_{<}=r \text { and } r_{>}=R, \text { whence } \\
& \frac{1}{|\mathbf{R}-\mathbf{r}|}=\frac{1}{R} \sum_{l=0}^{\infty}\left(\frac{r}{R}\right)^{l} P_{l}(\cos \omega)
\end{aligned}
$$

And,

$$
V(\mathrm{r})=Q \sum_{l} \frac{r^{l}}{R^{l+1}} \frac{4 \pi}{2 l+1} \sum_{m=-l}^{l} Y_{l m}(1) Y_{l m}^{*}(2),
$$

where (1) and (2) denote angular coordinates for the electron and ligand, respectively. Expanding,

$$
V(\mathrm{r})=Q / R\left\{1+\mathrm{r} \cdot \mathbf{R} / R^{2}+\frac{1}{2}\left[3(\mathrm{r} \cdot \mathbf{R})^{2}-r^{2} R^{2}\right] / R^{4}+\cdots\right\}
$$

where we use the phase convention of Condon and Shortley ${ }^{32}$ that $Y_{l_{m}}^{*}=(-1)^{m} Y_{t,-m}$. Since we are using only configurations with $s$ and/or $p$ electrons, terms in $V(\mathbf{r})$ higher than $l=2$ are not needed. If we have $N$ ligands

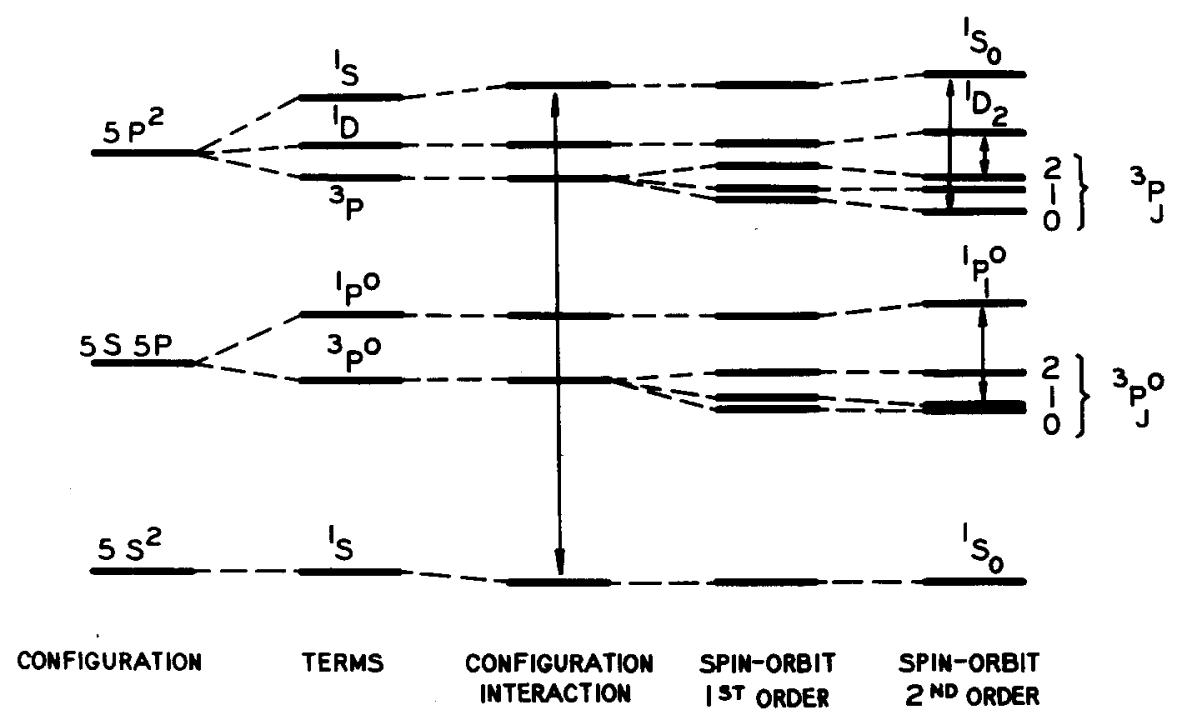

FIG. 1. Diagram showing the first-order electrostatic firstand second-order spin-orbit, and second-order electrostatic (configuration mixing) interactions for a gaseous $\mathrm{Xe}(+6)$ ion. 


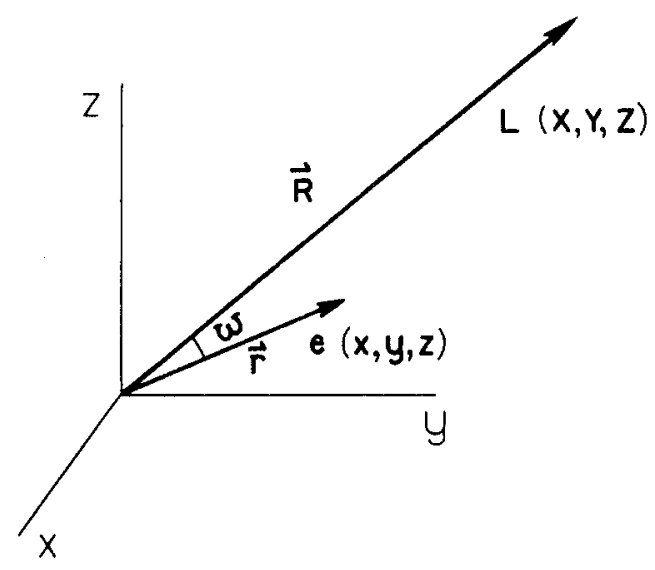

FIG. 2. Coordinate system for a ligand $L$ and an electron around a nucleus.

with charges $Q_{k}$ and a distance $R_{k}$, surrounding the central metal ion, with $n$ electrons, then the total potential energy is a sum over ligands and electrons:

$$
\begin{aligned}
V_{\mathrm{CF}} & =\sum_{i}^{n} V\left(\mathbf{r}_{i}\right) \\
& =\sum_{i}^{n} \sum_{k}^{N} \frac{Q_{k}}{R_{k}}\left\{1+\mathbf{r}_{i}^{0} \mathbf{R}_{k} / R_{k}^{2}+\cdots+\right\} .
\end{aligned}
$$

The geometry of the ligands is unspecified in Eq. (18). Since $V_{C F}$ is a one-electron operator and contains no spin-dependent terms, we only need to compute the matrix elements between one-electron orbitals with the same spin. Defining

$$
I\left(l_{1} m_{1} ; l_{2} m_{2}\right) \equiv\left\langle l_{1} m_{1}\left|V\left(r_{i}\right)\right| l_{2} m_{2}\right\rangle \text {. }
$$

We then obtain, using spherical harmonics as given by Condon and Shortley: ${ }^{32}$

$$
\begin{aligned}
& I(s, 0 ; s, 0)=\sum_{k}^{N} \frac{Q_{k}}{R_{k}} \\
& I(s, 0 ; p, 0)=\frac{1}{\sqrt{3}}\left\langle r_{i}\right\rangle_{s p} \sum_{k}^{N} \frac{Z_{k}}{R_{k}} Q_{k},
\end{aligned}
$$

where $\left\langle r_{i}\right\rangle_{s p}=\left\langle R_{s}\left|r_{i}\right| R_{p}\right\rangle$;

$$
\begin{aligned}
& I(s, 0 ; p, 1)=-\frac{1}{\sqrt{6}}\left\langle r_{i}\right\rangle_{s p} \sum_{k}^{N} \frac{X_{k}+i Y_{k}}{R_{k}^{3}} Q_{k}, \\
& I(s, 0 ; p, 1)=-I(s, 0 ; p,-1)^{*}, \\
& I(p, 0 ; p, 0)=\sum_{k}^{N}\left\{\frac{1}{R_{k}}+\frac{1}{5}\left\langle r_{i}^{2}\right\rangle_{p p} \frac{3 Z_{k}^{2}-R_{k}^{2}}{R_{k}^{5}}\right\} Q_{k},
\end{aligned}
$$

where $\left\langle r_{i}^{2}\right\rangle_{p p}=\left\langle R_{p}\left|r_{i}^{2}\right| R_{p}\right\rangle$;

$$
\begin{aligned}
& I(p, 0 ; p, 1)=-\frac{3}{5 \sqrt{2}}\left\langle r_{i}^{2}\right\rangle_{p p} \sum_{k}^{N} \frac{Z_{k}\left(X_{k}+i Y_{k}\right)}{R_{k}^{5}} Q_{k}, \\
& I(p, 0 ; p, 1)=-I(p, 0 ; p,-1)^{*}, \\
& I(p, \pm 1 ; p, \pm 1)=\sum_{k}^{N}\left\{\frac{1}{R_{k}}-\frac{1}{10}\left\langle r_{i}^{2}\right\rangle_{p p} \frac{3 Z_{k}^{c}-R_{k}^{2}}{R_{k}^{5}}\right\} Q_{k}, \\
& I(p, 1 ; p,-1)=-\frac{3}{10}\left\langle r_{i}^{2}\right\rangle_{p p} \sum_{k}^{N} \frac{\left(X_{k}-i Y_{k}\right)^{2}}{R_{k}^{5}} Q_{k} .
\end{aligned}
$$

In the above, the orbitals are denoted by $\left|l, m_{1}\right\rangle$, mean ing that complex components are chosen for the $p$ orbitals, with $X_{k}, Y_{k}, Z_{k}$ denoting the Cartesian coordinates of the $k$ th ligand, and the asterisk denoting a complex conjugate.

In summary, the effective Hamiltonian for the molecule is

$$
\begin{aligned}
H_{\text {total }}= & \sum_{i=1}^{2}\left(-\frac{1}{2} \nabla_{i}^{2}-\frac{Z}{r_{i}}\right)+\frac{1}{r_{12}} \\
& +\sum_{i=1}^{2} \xi\left(r_{i}\right) l_{i} \cdot \mathrm{s}_{i}+V_{\mathrm{CF}}+V_{\mathrm{tons}},
\end{aligned}
$$

where

$$
V_{\mathrm{ions}}=-\sum_{k=1}^{6} \frac{Z Q_{k}}{R_{k}}+\sum_{j<k} \frac{Q_{j} Q_{k}}{R_{j k}}
$$

is the previously undescribed electrostatic interaction among the $F^{-}$ligands and xenon +8 core, $R_{j k}=\left|\mathbf{R}_{j}-\mathbf{R}_{k}\right|$, $Q_{j}$ is the charge of the fluoride ligand $(-1)$ and $Z$ is the xenon core charge of +8 . We then evaluate the $H_{\mathrm{so}}$ matrix in the (ELSJ $\left.M_{J}\right)$ basis, transform to the ( $E L S M_{L} M_{S}$ ) basis, add to it the $V_{C F}$ matrix in that basis and the $H_{0}$ matrix, the latter readily evaluated in either basis, and finally add the diagonal matrix of $V_{\text {ions }}$ before diagonalizing the complete $28 \times 28$ matrix yielding eigenvalues and eigenvectors in the $\left(E L S M_{L} M_{S}\right)$ basis.

Before carrying out numerical calculations, we need a number of electronic parameters. These are the quantities $E_{\mathrm{Av}}(5 s, 5 s), E_{\mathrm{Av}}(5 s, 5 p)$, and $E_{\mathrm{Av}}(5 p, 5 p)$, the repulsion parameters $F_{1}(5 s, 5 p)$ and $F_{2}(5 p, 5 p)$, the spinorbit parameter $\zeta(5 p)$, and the radial integrals $\left\langle r_{i}\right\rangle_{s p}$ and $\left\langle r_{i}^{2}\right\rangle_{p p}$, the latter two being defined in Eq. (20). Since we are only interested in relative energies we define an effective energy "gap"

$$
\Delta E_{5 s-5 p} \equiv E_{\mathrm{A} v}(5 s, 5 p)-E_{\mathrm{Av}}\left(5 s^{2}\right)
$$

and then eliminate a parameter by assuming that

$$
E_{\mathrm{Av}}\left(5 p^{2}\right)-E_{\mathrm{Av}}\left(5 s^{2}\right)=2 \Delta E_{5 s-s p} \text {. }
$$

A useful empirical way to obtain nearly correct information on a given ion is to extrapolate from known properties of the ions of its isoelectronic series. We take the spectroscopic data ${ }^{37}$ for $\mathrm{Cd}(+0), \operatorname{In}(+1), \operatorname{Sn}(+2)$, $\mathrm{Sb}(+3)$, and $\mathrm{Te}(+4)$, which are isoelectronic to $\mathrm{Xe}(+6)$, and extrapolate (Fig. 3) to obtain the energy levels (Table II) for $\mathrm{Xe}(+6)$. No data for $\mathrm{I}(+5)$ were available.

From these extrapolated results for $\mathrm{Xe}(+6)$, we obtain

$$
\begin{aligned}
& F_{1}(5 s, 5 p)=17301 \mathrm{~cm}^{-1}=2.15 \mathrm{eV}, \\
& F_{2}(5 p, 5 p)=2560 \mathrm{~cm}^{-1}=0.32 \mathrm{eV},
\end{aligned}
$$

and,

$$
\zeta_{5 p} \approx 11000 \text { to } 16000 \mathrm{~cm}^{-1}=1.4-2.0 \mathrm{eV} .
$$

Note that from these, we have

$$
\begin{aligned}
\Delta E_{5 s-5 p}= & E_{\mathrm{Av}}(5 s, 5 p)-E_{\mathrm{Av}}\left(5 s^{2}\right)=118852 \mathrm{~cm}^{-1}=14.74 \mathrm{eV} \\
& E_{\mathrm{Av}}\left(5 p^{2}\right)-E_{\mathrm{Av}}\left(5 s^{2}\right)=245437 \mathrm{~cm}^{-1}=30.42 \mathrm{eV} .
\end{aligned}
$$

which agrees well with our assumption of Eq. (23).

It is well-known that in a complex ion term separa- 


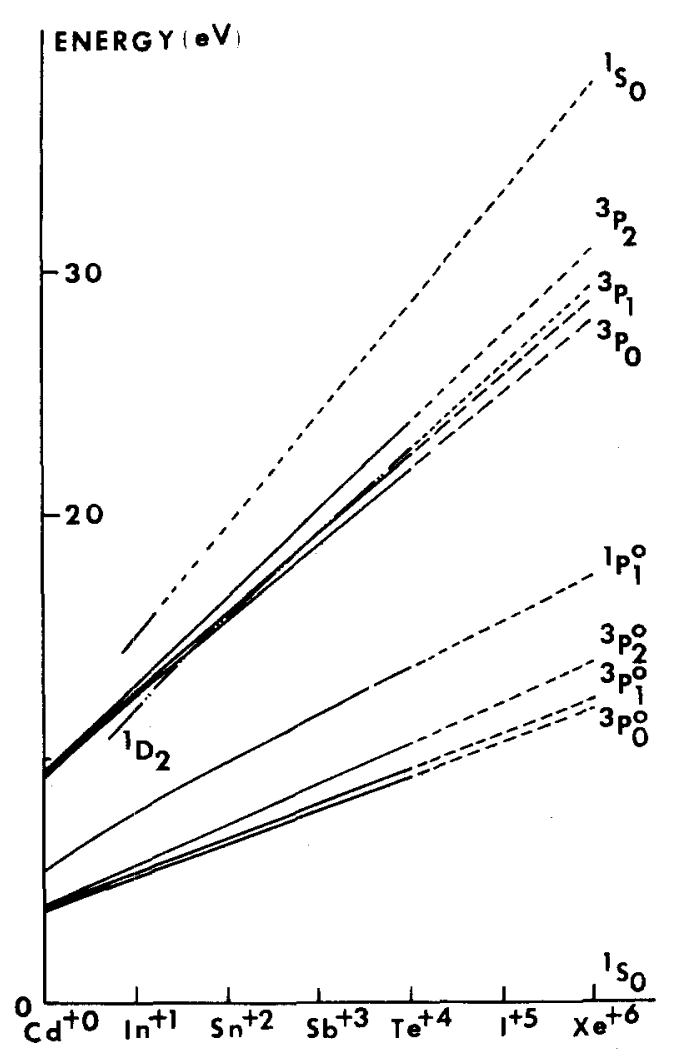

FIG. 3. Energy levels in electron volts for gaseous $\mathrm{Xe}(+6)$ ion as extrapolated (dashed lines) from observed levels (connected by solid lines) for isoelectronic ions. Data are from Ref. 37.

tions and spin-orbit coupling constants are both smaller than in the corresponding gaseous ion. ${ }^{38-42}$ It has been estimated by Walton, Mathews, and Jørgensen ${ }^{43}$ that for a complex ion with a $d^{10} s^{2}$ configuration that the radial wavefunction is so considerably changed that the spinorbit coupling constant in general will be decreased to about half and the electron repulsion parameters will be decreased to about a third of their corresponding values in the gaseous ion. A complete theory to explain and predict this reduction is yet to be formulated. However, we use these estimates as a rule of thumb, and study the trend of these parameters in those complexes isovalent to $\mathrm{XeF}_{6}$ to find a set of electronic parameters better suited to our study. They are listed in Table III.

TABLE II. Energy levels for $\mathrm{Xe}(+6)$ as extrapolated from energy levels of isoelectronic ions.

\begin{tabular}{lllc}
\hline \hline Configuration & Term & $J$ & Energy (eV) \\
\hline$\left(5 s^{2}\right)$ & ${ }^{1} S$ & 0 & 0.0 \\
$(5 s 5 p)$ & ${ }^{3} P^{0}$ & 0 & 12.4 \\
& & 1 & 13.2 \\
& & 2 & 14.4 \\
& ${ }^{1} P^{0}$ & 1 & 17.6 \\
$\left(5 p^{2}\right)$ & ${ }^{3} P$ & 0 & 28.1 \\
& & 1 & 29.0 \\
& & 2 & 31.1 \\
& ${ }^{1} D$ & 2 & 29.6 \\
& ${ }^{1} S$ & 0 & 37.8 \\
\hline \hline
\end{tabular}

TABLE III. $\Delta E_{5 s-5 p}$, Slater-Condon parameters and spinorbit coupling constants for ions and complexes of the $d^{10} s^{2}$ system.

\begin{tabular}{lcllll}
\hline \hline Ion/Complex & $\Delta E_{5 s-5 p}{ }^{\mathrm{a}}$ & $F_{1}^{\mathrm{a}}$ & $F_{2}^{\mathrm{a}}$ & $\zeta_{5 p}{ }^{\mathrm{a}}$ & Ref. \\
\hline $\mathrm{Xe}(0)$ & 39.57 & 2.23 & 0.22 & 0.51 & 44 \\
$\mathrm{Xe}(+2)$ & 11.78 & 1.10 & 0.27 & 0.80 & 37 \\
$\mathrm{Xe}(+6)$ & 14.74 & 1.91 & 0.32 & 1.7 & Extrapolation \\
$\mathrm{XeF}_{6}$ & 0.6 & & & & 45 \\
& 2.4 & & & & 46 \\
& 3.7 & & & & 47 \\
& 4.0 & 0.6 & & & 48 \\
$\mathrm{Te}^{(+4)}$ & 10.6 & 1.98 & & & 37 \\
$\mathrm{TeCl}_{6}^{-2}$ & 16.10 & 1.83 & & 0.90 & 37 \\
& 3.76 & 0.375 & & 0.55 & 49 \\
$\mathrm{TeBr}_{6}^{-2}$ & 3.54 & 0.40 & & & 39 \\
$\mathrm{Sb}^{+3}{ }^{-3}$ & 3.25 & 0.41 & & 0.4 & 49 \\
$\mathrm{SbCl}_{6}^{-3}$ & 9.46 & 1.627 & 0.82 & 0.67 & 37 \\
$\mathrm{SbBr}_{6}^{-3}$ & 4.9 & 0.57 & & 0.24 & 38,43 \\
$\mathrm{Sn}^{+2}+2$ & 3.7 & 0.57 & & & 43 \\
$\mathrm{SnCl}_{6}^{-4}$ & 7.8 & 1.41 & 0.55 & 0.47 & 37 \\
$\mathrm{SnBr}_{6}^{-4}$ & 3.06 & 0.55 & & & 50 \\
$\mathrm{SnI}_{2}$ & 4.27 & 0.56 & & & 47 \\
$\mathrm{SnI}_{6}^{-4}$ & 3.4 & 0.60 & & & 51 \\
$\left.\mathrm{In}^{+4}+1\right)$ & 3.7 & 0.40 & & & 39 \\
$\mathrm{InCl}_{6}^{-5}$ & 6.1 & 1.14 & 0.24 & 0.29 & 37 \\
$\mathrm{InBr}_{6}^{-5}$ & 4.9 & 0.53 & & & 39 \\
$\mathrm{InI}_{6}^{-5}$ & 4.4 & 0.31 & & & 39 \\
\hline \hline
\end{tabular}

${ }^{\text {aAll }}$ energy parameters are listed in electron volts.

Another parameter shown to be sensitive to the energy calculation is the $\Delta E_{5 s-5 p}$ of Eq. (22). The value for $\Delta E_{5 s-5 p}$ we have extrapolated for the $\mathrm{Xe}(+6)$ ion is about $15 \mathrm{eV}$, which is very high for the $\mathrm{XeF}_{6}$ molecule. It is also a general trend that in a complex ion $\Delta E_{n l-n^{\prime} l^{\prime}}$ will be less than in gaseous ion, ${ }^{38,39}$ as is the rule of thumb that for a set of given ligands the $\Delta E_{5 s-5 b}$ is rather constant in an isoelectronic series. ${ }^{40}$ (Table III) We also list the various values of $\Delta E_{5 s-5 p}$ for $\mathrm{XeF}_{6}$ used or implied by different authors. From these considerations, it is felt that the set of parameters $\left(\Delta E_{5 s-5 p}, F_{1}, F_{2}, \zeta_{5 p}\right)$ in electron volts which will best describe the molecule is around $(3.5,0.5,0.08,0.87)$, where we have obtained $F_{2}$ by scaling with the same numerical factor as with $F_{1}$. However, we have also used other parameter sets, with $\Delta E_{5 s-5 p}$ ranging from 0.5 to $3.5 \mathrm{eV}, F_{1}$ from 0.3 to $1.91 \mathrm{eV}, F_{2}$ from 0.05 to $0.32 \mathrm{eV}$, and $\zeta_{5 p}$ from 0.87 to $1.7 \mathrm{eV}$.

To obtain values of $\left\langle r^{2}\right\rangle_{p p}$ and $\langle r\rangle_{s p}$ we used the only available high-quality xenon radial wavefunctions, nameIy those for a neutral xenon atom as calculated by the Gaussian basis set self-consistent field (GTO-SCF) procedure. ${ }^{52}$ Fourteen $s$-type Gaussians and eleven $p$ type were used in the calculation. From these we obtain 


$$
\begin{aligned}
\left\langle r^{2}\right\rangle_{p p}^{1 / 2} & =\left\langle R_{5 p}\left|r^{2}\right| R_{5 p}\right\rangle^{1 / 2} \\
& =1.64954 \mathrm{a} . \mathrm{u} .
\end{aligned}
$$

and

$$
\begin{aligned}
\langle r\rangle_{s p} & =\left\langle R_{5 s}|r| R_{5 p}\right\rangle \\
& =2.05495 \mathrm{a} . \mathrm{u} .
\end{aligned}
$$

Although obtained from a neutral atom wavefunction, rather than one for $\mathrm{Xe}(+6)$, we think these values are a useful estimate, based as they are on a function which is radially expanded relative to $\mathrm{Xe}(+6)$. The same radial functions can be used to calculate the repulsion parameters, yielding an $F_{1}(5 s, 5 p)$ value of $2.23 \mathrm{eV}$ and an $F_{2}(5 p, 5 p)$ value of $0.22 \mathrm{eV}$ (Table III), both roughly comparable to the extrapolated $\mathrm{Xe}(+6)$ values, but decidedly greater than the scaled-down values.

\section{SYMMETRY CONSIDERATIONS FOR $\mathrm{XeF}_{6}$}

Though the gaseous $\mathrm{XeF}_{6}$ molecule is thought to be a distorted octahedron, our discussion of the symmetry of this molecule can begin with a consideration of it as a regular octahedron, then considering distortions which may lower the energy. With the particular model we are using there is no bond-stretching restoring force, so that we set a restriction that the six ligands $\left(F^{-}\right)$are only to move on the surface of a sphere with radius $R=1.89 \mathrm{~A}^{17}$ We therefore search for a pure bending mode which will change the geometry and stabilize the molecule; the stretching modes are of no particular interest to us here because: (1) they are, in general, energetically more expensive than pure bending, and (2) in the case of the $a_{1 g}$ mode, it does not at all change the molecular geometry. Thus, we want to examine the effects on $\mathrm{XeF}_{6}$ via pure bending part of $t_{1 u}, t_{2 u}$, and $t_{2 \xi} .{ }^{53}$ With the Hamiltonian as given by Eq. (21) if we distort the molecule away from $O_{h}$ along some symmetry coordinate the new Hamiltonian can be written as a Taylor's expansion about $O_{h}$ as $5^{54-57}$

$$
H=H_{0_{h}}+\left(\frac{\delta H}{\delta Q}\right)_{0_{h}} Q+\frac{1}{2}\left(\frac{\delta^{2} H}{\delta Q^{2}}\right)_{0_{h}} Q^{2}+\frac{1}{6}\left(\frac{\delta^{3} H}{\delta Q^{3}}\right)_{0_{h}} Q^{3}+\cdots,
$$

where $Q$ is the symmetry coordinate displacement and

$$
\begin{aligned}
E_{0}= & E_{0}^{0}+\left\langle 0\left|\frac{\delta H}{\delta Q}\right| 0\right\rangle Q+\left\{\frac{1}{2}\left\langle 0\left|\frac{\delta^{2} H}{\delta Q^{2}}\right| 0\right\rangle-\sum_{i}^{\prime} \frac{\langle i|\delta H / \delta Q| 0\rangle^{2}}{E_{i}^{0}-E_{0}^{0}}\right\} Q^{2} \\
& +\left\{\sum _ { j } ^ { \prime } \left[\sum_{i}^{\prime}\left(\frac{\langle 0|\delta H / \delta Q| j\rangle\langle j|\delta H / \delta Q| i\rangle\langle i|\delta H / \delta Q| 0\rangle}{\left(E_{i}^{0}-E_{0}^{0}\right)\left(E_{j}^{0}-E_{0}^{0}\right)}\right)-\frac{\langle 0|\delta H / \delta Q| 0\rangle\langle 0|\delta H / \delta Q| j\rangle\langle j|\delta H / \delta Q| 0\rangle}{E_{j}^{0}-E_{0}^{0}}\right.\right. \\
& \left.\left.-\frac{\langle 0|\delta H / \delta Q| j\rangle\left\langle j\left|\delta^{2} H / \delta Q^{2}\right| 0\right\rangle}{E_{j}^{0}-E_{0}^{0}}\right]+\frac{1}{6}\left\langle 0\left|\frac{\delta^{3} H}{\delta Q^{3}}\right| 0\right\rangle\right\} Q^{3}+0\left(Q^{4}\right)+\cdots
\end{aligned}
$$

In general, from Eq. (25), the stabilization of the molecule comes from the second-order Jahn-Teller effect (or, pseudo-Jahn-Teller effect), as the first-order term vanishes except in true Jahn-Teller cases. Therefore we must have the term

$$
\sum_{i}^{\prime} \frac{|\langle i|\delta H / \delta Q| 0\rangle|^{2}}{E_{i}^{0}-E_{0}^{0}}
$$

be important; that is

$$
\langle i|\delta H / \delta Q| 0\rangle \neq 0 \text { for some }|i\rangle \text {, and }|i\rangle
$$

lying close to $|0\rangle .56,57$

We list below the symmetry species for the different states of $\mathrm{XeF}_{6}$ assuming an $O_{h}$ symmetry as

$$
\begin{gathered}
5 p^{2}\left\{\begin{array}{l}
{ }^{1} S_{0} \rightarrow A_{1 g} \\
{ }^{1} D_{2} \rightarrow T_{2 g}, E_{g} \\
{ }^{3} P_{2} \rightarrow T_{2 g}, E_{g} \\
{ }^{3} P_{1} \rightarrow T_{1 g} \\
{ }^{3} P_{0} \rightarrow A_{1 g}
\end{array}\right. \\
\left\{\begin{array}{l}
{ }^{1} P^{0} \rightarrow T_{1 u} \\
{ }^{3} P_{2}^{0} \rightarrow T_{2 u}, E_{u} \\
{ }^{3} P_{1}^{0} \rightarrow T_{1 u} \\
{ }^{3} P_{0}^{0} \rightarrow A_{1 u}
\end{array}\right.
\end{gathered}
$$

$$
5 s^{2} \quad\left\{{ }^{1} S_{0} \rightarrow A_{1 g} .\right.
$$

Without spin-orbit coupling, the correspondances are

$$
\begin{aligned}
& { }^{1} S-{ }^{1} A_{1 g},{ }^{1} D \rightarrow\left({ }^{1} T_{2 g},{ }^{1} E_{g}\right),{ }^{3} P-{ }^{3} T_{1 g}, \\
& { }^{1} P^{0} \rightarrow{ }^{1} T_{1 u}, \text { and }{ }^{3} P^{0} \rightarrow{ }^{3} T_{1 u} .
\end{aligned}
$$

It is easy to show by symmetry that the only excited state connected to the ${ }^{1} S\left({ }^{1} A_{1 g}\right)$ ground state is the ${ }^{1} P^{0}\left({ }^{1} T_{1 u}\right)$ state, with this state being connected by the $t_{1 u}$ bending and stretching vibrations. To the extent that spin-orbit coupling is important, this conclusion must be modified to take into account additional mixings that are readily seen from $(26)$ to be with ${ }^{3} P_{1}^{0}\left(T_{1 u}\right)$ by $t_{1 u}$ vibrations, ${ }^{3} P_{2}^{0}\left(T_{2 u}\right)$ by the $t_{2 u}$ bending mode, ${ }^{1} D_{2}$ and ${ }^{3} D_{2}$ (both $T_{2 g}$ ) by the $t_{2 g}$ bending mode, and ${ }^{1} D_{2}$ and ${ }^{3} P_{2}$ (both $E_{g}$ ) by the $e_{g}$ stretching mode, which we ignore.

The symmetry coordinates $Q_{t_{1 u}}, Q_{t_{2 u}}$ and $Q_{t_{2 g}}$ we use are of the following forms.

$$
\begin{aligned}
& Q_{t_{1,}, a}(\text { bend) } \\
& =\frac{1}{\sqrt{8}} \gamma_{\mathrm{X}-\mathrm{F}}\left(\alpha_{24}+\alpha_{34}+\alpha_{45}+\alpha_{46}-\alpha_{12}-\alpha_{13}-\alpha_{15}-\alpha_{16}\right), \\
& Q_{t_{1 u^{\prime}}} \text { (bend) } \\
& =\frac{1}{\sqrt{8}} r_{\mathrm{Xe}-\mathrm{F}}\left(\alpha_{15}+\alpha_{35}+\alpha_{45}+\alpha_{56}-\alpha_{12}-\alpha_{23}-\alpha_{24}-\alpha_{26}\right), \\
& Q_{t_{1, u}, c}(\text { bend) } \\
& =\frac{1}{\sqrt{8}} r_{\mathrm{XQ}-\mathrm{F}}\left(\alpha_{16}+\alpha_{26}+\alpha_{46}+\alpha_{56}-\alpha_{13}-\alpha_{23}-\alpha_{34}-\alpha_{35}\right) \text {, }
\end{aligned}
$$




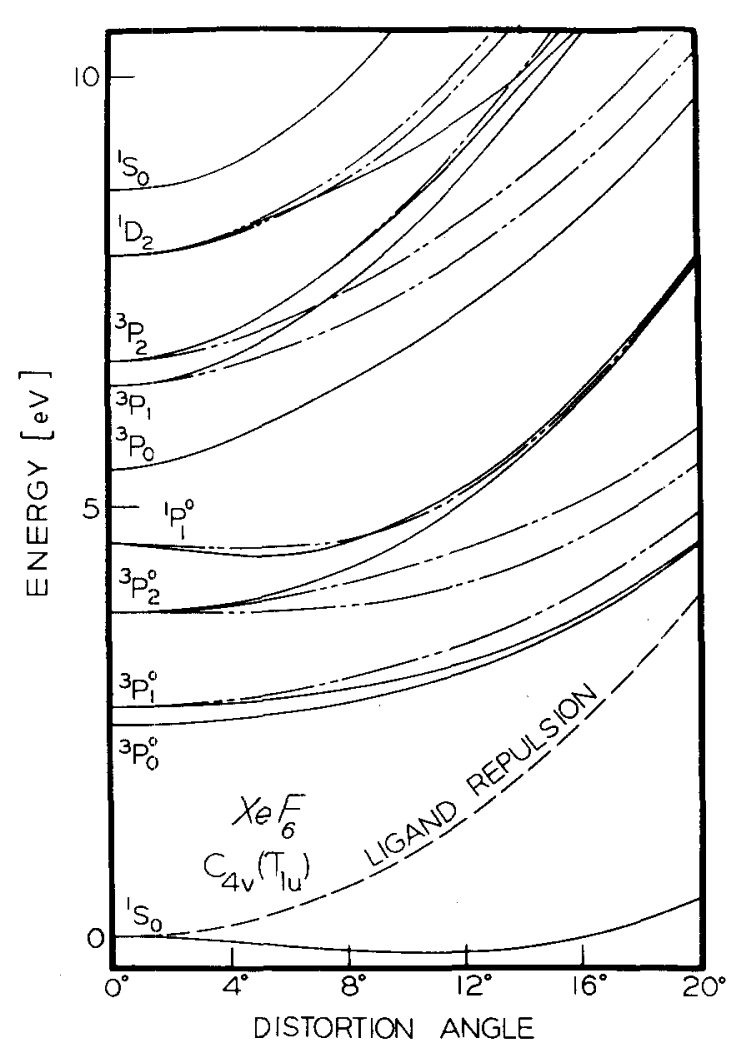

FIG. 4. Electronic energy in electron volts vs the $t_{1 u}$ bending angle $\Delta$ for the set of electronic parameters $\Delta E_{5 s m 5 p}=3.5$, $F_{1}=0.5, F_{2}=0.08$, and $\zeta_{5 p}=0.87 \mathrm{eV}$. The molecular symmetry is $C_{4 v}$ except at the origin, where it is $O_{h}$. Calculations were made at $\Delta=0^{\circ}, 2^{\circ}, 4^{\circ}, 8^{\circ}, 12^{\circ}, 16^{\circ}$, and $20^{\circ}$, with solid lines denoting nondegenerate states and dashed lines denoting doubly degenerate states.

$$
\begin{aligned}
& Q_{t_{2 u^{\prime}} a} \\
& =\frac{1}{\sqrt{8}} \gamma_{\mathrm{Xe}-\mathrm{F}}\left(\alpha_{12}-\alpha_{13}+\alpha_{15}-\alpha_{16}-\alpha_{24}+\alpha_{34}-\alpha_{45}+\alpha_{46}\right), \\
& Q_{t_{2, u}, b} \\
& =\frac{1}{\sqrt{8}} \gamma_{\mathrm{Xe}-\mathrm{F}}\left(\alpha_{15}-\alpha_{35}+\alpha_{45}-\alpha_{56}-\alpha_{12}+\alpha_{23}-\alpha_{24}+\alpha_{26}\right), \\
& Q_{t_{2 u^{\prime}}, c}=\frac{1}{\sqrt{8}} r_{X \theta-F}\left(\alpha_{13}-\alpha_{23}+\alpha_{34}-\alpha_{35}-\alpha_{16}+\alpha_{26}-\alpha_{46}+\alpha_{56}\right) \\
& Q_{t_{2 g^{\prime}} \mathrm{c}}=\frac{1}{2} r_{\mathrm{XO}-\mathrm{F}}\left(\alpha_{26}+\alpha_{35}-\alpha_{23}-\alpha_{56}\right) \text {, } \\
& Q_{t_{2, b}, b}=\frac{1}{2} r_{\mathrm{XE}-\mathrm{F}}\left(\alpha_{16}+\alpha_{34}-\alpha_{13}-\alpha_{46}\right) \text {, } \\
& Q_{t_{2 k}, c}=\frac{1}{2} r_{\mathrm{X}-F}\left(\alpha_{15}+\alpha_{24}-\alpha_{12}-\alpha_{45}\right) \text {, }
\end{aligned}
$$

where $\alpha_{i j}=<F(i)-\mathrm{Xe}-\mathrm{F}(j)$.

For $O_{h}$ symmetry, the $F$ atoms are located on the axes of a right-handed Cartesian coordinate system, with atoms 1 and 4 on $\pm x, 2$ and 5 on $\pm y$, and 3 and 6 on $\pm z$, respectively.

\section{POTENTIAL ENERGY CURVES FOR $T_{1 u}$ BENDING}

We set the conditions for the $t_{1 u}$ mode of bending such that: (1) $r_{\mathrm{X}-\mathrm{F}}=1.89 \times 10^{-8} \mathrm{~cm}$, and (2) $\alpha_{15}=\alpha_{35}=\cdots=\Delta$. In Fig. 4, we plot the calculated electronic energies vs. deformation angle $\Delta$ for a structure of symmetry $C_{4 v}$. In Table IV we list the position of the minimum and the lowering of the energy of the ground state for different parameters used for this case of $C_{4 v}\left(t_{1 u}\right)$.

The $t_{1 u}$ (bending) deformation is geometrically equivalent to a "lone pair electrons" pushing the ligands aside and protruding into the coordination sphere. ${ }^{17,31}$ It can lead to $C_{4 v}, C_{3 v}$, and $C_{2 v}$ symmetries (Fig. 5). We note that as the numerical value of the $\Delta E_{5 s-5 p}$ parameter decreases, the pseudo-Jahn-Teller effect becomes more and more significant (Table IV); this may be described as an increase in the $p$ character of the lone pair. Decreases in the other parameters are seen (Table III) to have a similar effect. Note that also a maximum shifting of the minimum of the ground state energy curve is reached where the angular deformation is about $14^{\circ} \sim 15^{\circ}$ as we change the electronic parameters (in the case of $C_{4 v}$ ). This appears to indicate a limiting effect on the lone pair protruding into the coordination sphere. For the assumed best set of parameters $(3.5,0.5,0.08,0.87$ $\mathrm{eV})$, the ground state energy minimum occurs at $11^{\circ}$ for the deformation angle $\Delta$ with a lowering of energy of about $0.204 \mathrm{eV}$ or $1604 \mathrm{~cm}^{-1}$ for $C_{4 v}$ symmetry; at about $8.9^{\circ}(\delta)$ with lowering of energy about $0.225 \mathrm{eV}$ or about $1800 \mathrm{~cm}^{-1}$ for $C_{3 v}$ symmetry; and at about $7.8^{\circ}(\alpha)$ with lowering of energy about $0.220 \mathrm{eV}$ or about $1760 \mathrm{~cm}^{-1}$ for $C_{2 v}$ symmetry. (For the meaning of $\Delta, \delta$, and $\alpha$ see Fig. 5.) This is to be compared with the $t_{1 u}$ bending frequency $\nu_{4}$ of other hexafluorides ${ }^{6,58}$ : e.g. , TeF 6 , $v_{4}=325 \mathrm{~cm}^{-1}$, with an estimated reduced mass for the $t_{1 u}$ bending vibration of $8.6 \times 10^{-23} \mathrm{~g}$-molecule ${ }^{-1}$, which implies $\left\langle x^{2}\right\rangle^{1 / 2}=0.032 \mathrm{~A}$ or a root-mean-square angular displacement of about $1.0 \mathrm{deg}$; and $\mathrm{UF}_{6}, v_{4}=184 \mathrm{~cm}^{-1}$ (the softest $t_{1 u}$ bending frequency of known regular hexafluorides), with an estimated reduced mass for the $t_{1 u}$ vibration of $9.9 \times 10^{-23} \mathrm{~g}$-molecul $\mathrm{e}^{-1}$, which implies bending vibration of $9.9 \times 10^{-23} \mathrm{~g}-$ molecule $\mathrm{e}^{-1}$, which implies $\left\langle x^{2}\right\rangle^{1 / 2}=0.039 \mathrm{~A}$ or a root-mean-square angular displacement of about $1.1 \mathrm{deg}$. This indicates the nature of the distortion of $\mathrm{XeF}_{6}$ from $O_{h}$ via $t_{1 u}$ bending is not a simple fluctuation of geometry due to vibration.

TABLE IV. Ground state energy for various electronic parameters for $t_{1 u}$ pure bending $\left(C_{4 v}\right)$.

\begin{tabular}{lcl}
\hline \hline $\begin{array}{l}\Delta\left(E_{5 s-5 p}, F_{1}, F_{2}, \zeta_{5 p}\right), \\
(\text { all in eV) }\end{array}$ & $\begin{array}{l}\text { Position of } \\
\text { energy min } \\
(\Delta \mathrm{deg})\end{array}$ & $\left(E-E_{0_{h}}\right)(\mathrm{eV})$ \\
\hline$(3.5,1.91,0.32,1.7)$ & 0.0 & $\cdots$ \\
$(3.5,1.0,0.17,0.87)$ & $($ Very flat $)$ & $\ldots$ \\
$(3.5,0.7,0.11,0.87)$ & 10.0 & 0.085 \\
$(3.5,0.5,0.08,0.87)$ & 11.0 & 0.204 \\
$(3.5,0.3,0.05,0.87)$ & 12.0 & 0.340 \\
$(2.0,1.91,0.32,1.7)$ & 0.0 & $\ldots$ \\
$(2.0,1.91,0.17,0.87)$ & 10.5 & 0.136 \\
$(2.0,0.7,0.11,0.87)$ & 13.2 & 0.432 \\
$(2.0,0.5,0.08,0.87)$ & 14.0 & 0.680 \\
$(2.0,0.3,0.05,0.87)$ & 14.0 & 0.952 \\
$(1.0,1.91,0.32,1.7)$ & 4.7 & 0.017 \\
$(1.0,1.0,0.17,0.87)$ & 12.0 & 0.303 \\
$(1.0,0.7,0.11,0.87)$ & 14.3 & 0.714 \\
$(1.0,0.5,0.08,0.87)$ & 14.8 & 1.088 \\
$(1.0,0.3,0.05,0.87)$ & 15.0 & 1.461 \\
$(0.5,1.91,0.32,1.7)$ & 3.0 & 0.007 \\
$(0.5,1.0,0.17,0.87)$ & 10.0 & 0.262 \\
$(0.5,0.7,0.11,0.87)$ & 14.6 & 0.595 \\
$(0.5,0.5,0.08,0.87)$ & 14.9 & 0.918 \\
$(0.5,0.3,0.05,0.87)^{\mathrm{b}}$ & 14.5 & 1.258 \\
\hline \hline
\end{tabular}

${ }^{a} Q_{t_{1 u^{\prime}},}(\min )=2 \sqrt{ } 2\left(r_{\mathrm{Xe}-\mathrm{F}} \pi / 180\right) \Delta$.

${ }^{\mathrm{b}}$ The ground state in this case is ${ }^{3} P_{0}^{0}$ instead of ${ }^{1} S_{0}$. 

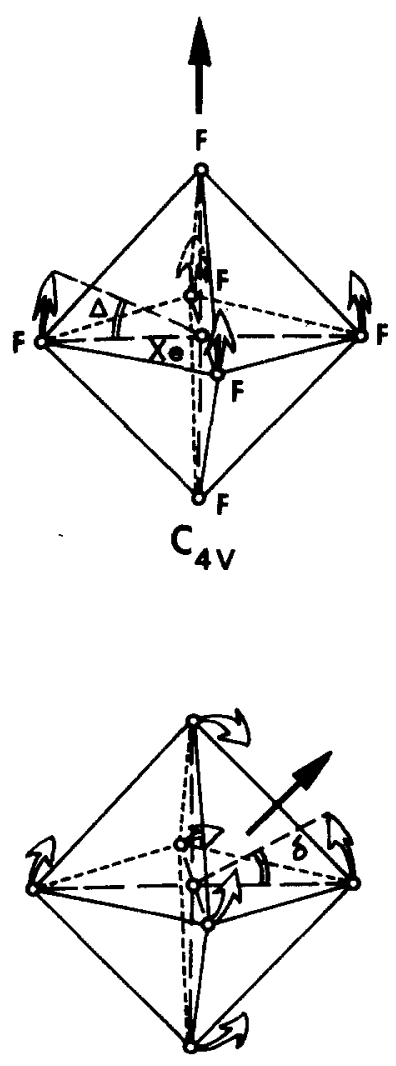

FIG. 5. Symmetries derived from $t_{1 u}$ bending vibration of an octahedral molecule. The deformation angles $\Delta$ for $C_{4 v}, \delta$ for $C_{3 v}$, and $\alpha$ and $\beta$ for $C_{2 v}$ are related in Eq. (35) for the case of constant $t_{1 u}$ radial displacement.
$\mathrm{C}_{3 \mathrm{~V}}$

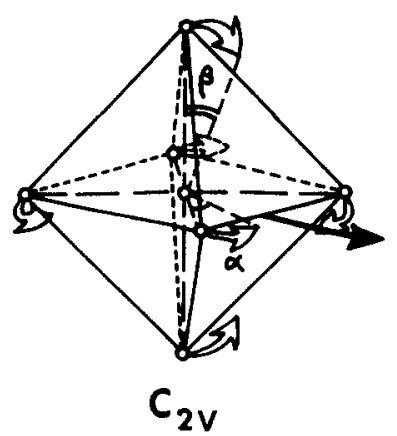

In the case of $C_{4 v}$, when the assumed best set of parameters is used, the ground state energy curve shows a negative force constant of about $1.0 \mathrm{mdyn} / \mathrm{A}$ at the $O_{h}$ origin. In the approximate form of a (one-dimensional) double-well vibrational potential, this energy curve near its minimum has a zero-point energy of : about $51 \mathrm{~cm}^{-1}$, with the first vibrational spacing being about $109 \mathrm{~cm}^{-1}$. In Fig. 6 we plot the ${ }^{1} A_{18}$ ground state energy curve as a function of $\Delta E_{5 s-5 p}$. Potential energy curves for all of the states for the $C_{3 v}$ structures are not shown as they are virtually identical to Fig. 4 when plotted on the compressed energy scale of the latter figure.

Let us now construct a $t_{1 u}$ bending space with the spherical polar coordinates

$$
\begin{aligned}
& R=\left[Q_{t_{1 u^{, a}}}^{2}+Q_{t_{1 u^{\prime} b}}^{2}+Q_{t_{1 u^{\prime}},}^{2}\right]^{1 / 2} \\
& \Theta=\cos ^{-1}\left[Q_{t_{1 u^{\prime}},} /\left(Q_{t_{1 u^{\prime}, a}}^{2}+Q_{t_{1 u}, b}^{2}+Q_{t_{1 u}, c}^{2}\right)^{1 / 2}\right]
\end{aligned}
$$

$$
\phi=\tan ^{-1}\left(Q_{t_{1 u^{, b}}} / Q_{t_{1 u^{, a}}}\right) \text {. }
$$

These coordinates are useful as the movement of the $\mathrm{XeF}_{6}$ molecule in the $t_{1 u}$ vibrational space at constant $R$ may be considered as a pseudorotation, passing through six $C_{4 v}$, eight $C_{3 v}$, and twelve $C_{2 v}$ structures, these corresponding to the faces, corners, and edges of a cube. With the assumed best set of parameters, the energies of the three structures relative to the $O_{n}$ symmetry structure were previously given as $-0.204,-0.225$, and $-0.220 \mathrm{eV}$ for $C_{4 v}, C_{3 v}$, and $C_{2 v}$, respectively. Thus relative to the $C_{3 v}$ minimum the $C_{4 v}$ energy is 0.021 $\mathrm{eV}$, while the $C_{2 v}$ energy is $0.005 \mathrm{eV}$. In Fig. 7 we show the orthogonal projection of the equipotential lines from the $\langle 100\rangle$ axis onto the [011] plane in the $t_{1 u}$ space. These results show that the energetically most favored paths are those which go through any of the $C_{3 v} \rightarrow C_{2 v} \rightarrow C_{3 v}$ $\leftarrow C_{2 v} \leftrightarrow \cdots$. Taking this path, if the frequency is large enough, and if the "rotation" is such that it goes through a greatest "circle" on this sphere in $t_{1 u}$ space; then an average effect of "zero dipole moment" from the lone pair is expected for the molecule. If the reduced mass of $\mathrm{XeF}_{6}$ in $t_{14}$ vibrational motion is estimated to be 8.7 $\times 10^{-23} \mathrm{~g}$-molecule ${ }^{-1}$, with the sphere corresponding to the energy minimum, i. e., $R=1.026 \AA$, then a rotational frequency of $2 B_{e} \sim 0.61 \mathrm{~cm}^{-1}$ is to be expected. It is of interest to observe that the following relationship exists among the ground state energies of different symmetries:

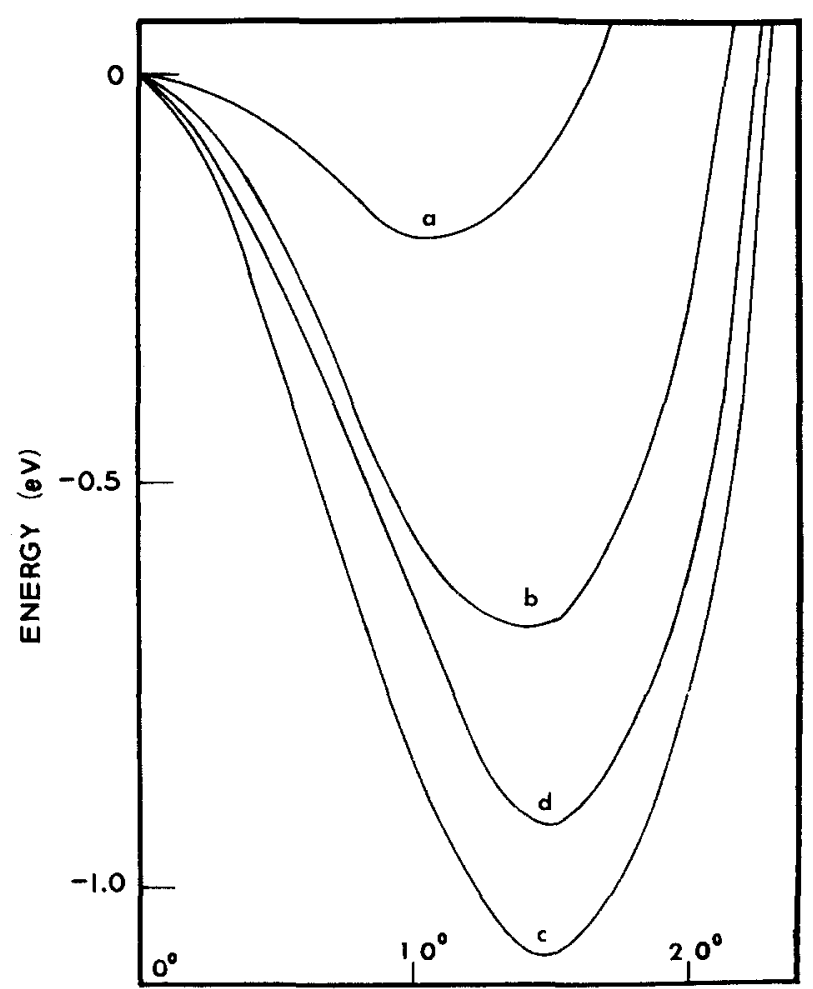

FIG. 6. Ground-state energy in electron volts vs $t_{1 u}$ bending angle $\Delta\left(C_{4 v}\right.$ symmetry $)$ as a function of the energy parameter $\Delta E_{5 s-5 p}$, this having values of (a) 3.5 , (b) 2.0 , (c) 1.0 , and (d) $0.5 \mathrm{eV}$. The $F_{2}, F_{1}$, and $\zeta$ parameters are $0.5,0.08$, and $0.87 \mathrm{eV}$, respectively, in each case. 


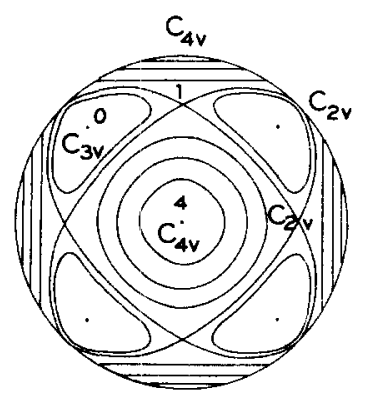

FIG. 7. Projection of equipotential lines for the groundstate energy from the $\langle 100\rangle t_{1 u}$ axis onto the [011] plane. The $C_{4 v}$ structure is at an energy of four arbitrary units relative to $C_{3 v}$, with the $C_{2 v}$ structure being at one unit relative to $C_{3 v}$.

$$
\begin{array}{r}
{\left[E_{0}\left(C_{4 v}\right)-E_{0}\left(C_{3 v}\right)\right] /\left[E_{0}\left(C_{2 v}\right)-E_{0}\left(C_{3 v}\right)\right]} \\
=0.021 \mathrm{eV} / 0.005 \mathrm{eV} \approx 4 / 1 .
\end{array}
$$

This result does not arise from the ligand repulsion, which on the contrary, slightly favored the $C_{4 v}$ structure by only $0.00046 \mathrm{eV}$ over the $C_{3 v}$ structure (see Fig. 8) but rather comes from the variation in the $s p$ mixing as the angular coordinates of Eq. (28) are changed.

The pseudorotational motion can be described as the motion of a particle on the surface of a sphere of fixed radius, but subject to a cubic potential energy. The potential energy $V$, with $R$ of Eq. (28) held constant, can be written as

$$
V(X, Y, Z)=X^{4}+Y^{4}+Z^{4}+\lambda\left(X^{2}+Y^{2}+Z^{2}-R^{2}\right)
$$

where $X=Q_{t_{1 u^{\prime}} a}, \quad Y=Q_{t_{1 u}, b}, Z=Q_{t_{1 u}, c}$ or in polar coor dinates,

$$
V(R, \Theta, \phi)=R^{4}\left(\sin ^{4} \phi \sin ^{4} \theta+\cos ^{4} \phi \sin ^{4} \theta+\cos ^{4} \theta\right) .
$$

There are readily shown ${ }^{17}$ to be six maxima with $C_{4 v}$ symmetry and an energy of one unit, 12 saddle points with $C_{2 v}$ symmetry and an energy of one-half unit, and eight minima of $C_{3 v}$ symmetry with an energy of onethird unit. (Fig. 7)

Therefore,

$$
\left[E\left(C_{4 v}\right)-E\left(C_{3 v}\right)\right] /\left[E\left(C_{2 v}\right)-E\left(C_{3 v}\right)\right]=4 / 1 .
$$

showing that our numerical results can be adequately described by Eq. (30) for the case of constant $R$ in $t_{1 u}$ bending space.

\section{PERTURBATION THEORY ANALYSIS OF $s-p$ MIXING IN $t_{1}$, BENDING SPACE}

For an $s$ orbital and a $p$ orbital situated in a field $V$ generated by $n$ point charges of $Q_{n}=-1$ for all $n$ arbitrarily located at $R_{n}\left(X_{n}, Y_{n}, Z_{n}\right)$, we have the interaction matrix elements as in Eqs. (18) and (20). If we are to restrict ourselves to the case of pure bending, where the point charges are only to move on the surface of a sphere of set radius [this does not imply that $R$ of Eq. (30) is constant, but merely that all $\mathrm{Xe}-\mathrm{F}$ bond lengths are held constant], it is convenient to make the following simplification and substitutions:

$$
\begin{aligned}
& R_{n}=1 \text { for all } n . \\
& \langle r\rangle_{s p}=a<1, \\
& \left\langle r^{2}\right\rangle_{p p}=b<1,
\end{aligned}
$$

$$
\begin{aligned}
& X_{n}=\sin \theta_{n} \cos \phi_{n}, \\
& Y_{n}=\sin \Theta_{n} \sin \phi_{n}, \\
& Z_{n}=\cos \Theta_{n},
\end{aligned}
$$

Thus, we can write the matrix elements as

$$
\begin{aligned}
& \langle s, 0|V| s, 0\rangle=n, \\
& \langle p, 0|V| p, 0\rangle=n+(b / 5) \sum_{k}\left(3 \cos ^{2} \Theta_{k}-1\right), \\
& \langle p, 1|V| p, 1\rangle=n-(b / 10) \sum_{k}\left(3 \cos ^{2} \Theta_{k}-1\right), \\
& \langle s, 0|V| p, 0\rangle=a / 3^{1 / 2} \sum_{k} \cos \Theta_{k} \\
& \langle s, 0|V| p, 1\rangle=a / 6^{1 / 2} \sum_{k} \sin \Theta_{k}\left(\cos \phi_{k}+i \sin \phi_{k}\right), \\
& \langle p, 0|V| p, 1\rangle=-3 b / 10(2)^{1 / 2} \sum_{k} \sin 2 \Theta_{k}\left(\cos \phi_{k}+i \sin \phi\right), \\
& \langle p, 1|V| p,-1\rangle=-(3 b / 10) \sum \sin ^{2} \Theta_{k}\left(\cos 2 \phi_{k}-i \sin 2 \phi_{k}\right) .
\end{aligned}
$$

In $t_{1 u}$ space, if we are considering the sphere defined by constant $R$ of Eq. (28), we have the following relationship:

$$
\Delta=(3 / 2)^{1 / 2} \delta=2^{1 / 2} \alpha=\beta,
$$

or

$$
\sin \Delta=\sin \left[(3 / 2)^{1 / 2} \delta\right]=\sin \left(2^{1 / 2} \alpha\right)=\sin \beta,
$$

where $\Delta$ is the angular displacement from $O_{h}$ in the case of $C_{4 v}$ symmetry, $\delta$ that in the case of $C_{3 v}$, and $\alpha$ and $\beta$ in the case of $C_{2 v}$ [Fig. (6)]. Using the power series for the sine function, we write in general

$$
\sin x=\frac{1}{a} \sin (a x)-\frac{(a x)^{3}\left(1-a^{2}\right)}{a^{3} 3 !}+\frac{(a x)^{5}\left(1-a^{4}\right)}{a^{5} 5 !}-\cdots+,
$$

or for the above variables

$$
\sin \delta=(2 / 3)^{1 / 2} \sin \Delta+(2 / 3)^{3 / 2} \Delta^{3} / 12-\cdots+
$$

and

$$
\sin \alpha=2^{-1 / 2} \sin \Delta+2^{-3 / 2} \Delta^{3} / 6 \cdots \cdots+.
$$

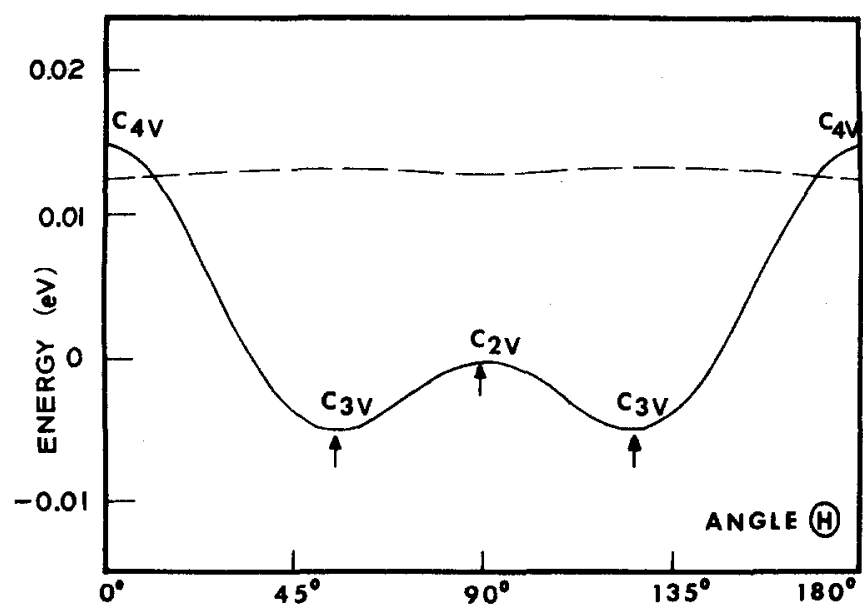

FIG. 8. Ground-state energy in electron volt vs $t_{1 u}$ pseudorotational angle $\Theta$. The dashed line shows the variation in the ligand-ligand repulsion energy. 
For sufficiently small displacements,

$$
\sin \Delta=(3 / 2)^{1 / 2} \sin \delta=2^{1 / 2} \sin \alpha=\sin \beta .
$$

However a careful analysis requires the extra terms in Eqs. (38), (39).

Now, subjecting the electrons to a field $V$ formed by six point charges, we consider the following cases.

A. Splitting of $p$ orbital alone, assuming Eq. (40)

1. $C_{4 v}$

$$
\begin{aligned}
& \langle p, 0|V| p, 0\rangle=6+12 b \sin ^{2} \Delta / 5, \\
& \langle p, 0|V| p, 1\rangle=6-6 b \sin ^{2} \Delta / 5,
\end{aligned}
$$

all other matrix elements are zero. Setting the energy of the state when the symmetry is $O_{h}$ to zero, then we have eigenvalues

$$
\begin{array}{ll}
\lambda_{1}=+(12 b / 5) \sin ^{2} \Delta . & \text { ( singlet) } \\
\left.\lambda_{2,3}\right)=-(6 b / 5) \sin ^{2} \Delta . & \text { (doublet) }
\end{array}
$$

2. $C_{3 v}$

$$
\begin{aligned}
\langle p, 0|V| p, 0\rangle & =+6 b \sin ^{2} \delta / 5, \\
& =+4 b \sin ^{2} \Delta / 5, \\
\langle p, 1|V| p, 1\rangle & =-3 b \sin ^{2} \delta / 5, \\
& =-2 b \sin ^{2} \Delta / 5,
\end{aligned}
$$

where the approximation of Eq. (40) is used for $\sin \Delta$ and all other matrix elements are zero. Therefore, the eigenvalues are

$$
\begin{array}{ll}
\lambda_{1}=+(4 b / 5) \sin ^{2} \Delta . & \text { (singlet) } \\
\lambda_{2,3}=-(2 b / 5) \sin ^{2} \Delta . & \text { (doublet) }
\end{array}
$$

3. $C_{2 v}$

$$
\begin{aligned}
& \langle p, 0|V| p, 0\rangle=-6 b \sin ^{2} \beta / 5=-6 b \sin ^{2} \Delta / 5, \\
& \langle p, 1|V| p, 1\rangle=3 b \sin ^{2} \beta / 5=3 b \sin ^{2} \Delta / 5, \\
& \langle p, 1|V| p,-1\rangle=i 3 b \sin ^{2} \beta / 5=i 3 b \sin ^{2} \Delta / 5,
\end{aligned}
$$

Therefore, to second-order the mostly $s$ ground state energy is

$$
E\left(C_{4 v}\right)=-\left(16 a^{2} \sin ^{2} \Delta / 3 U\right)\left\{1-\left(12 b \sin ^{2} / 5 U\right)\right\} .
$$

where no approximation is needed and all the other matrix elements are zero.

The eigenvalues are

$$
\begin{array}{ll}
\lambda_{1}=-(6 b / 5) \sin ^{2} \Delta . & \text { (singlet) } \\
\lambda_{2}=0, & \text { (singlet) } \\
\lambda_{3}=(6 b / 5) \sin ^{2} \Delta . & \text { (singlet) }
\end{array}
$$

We summarize the above results in Fig. 9. These results represent the variation in the splitting of the $p$ orbital by the variation of the strength $l=2$ term in the crystal field as the direction of bending in $t_{1 u}$ space is changed at constant $R$ (constant magnitude of $t_{1 u}$ bending). Note that this variation arises even when Eq. (40) is used. The terms from Eqs. (38), (39) that should be added contribute a term in $\Delta^{3} \sin \Delta$, which is of the order of $\Delta^{4}$, and even higher terms in $\Delta^{6}$, all of which are ignored here. However for the angular variation in the $s-p$ mixing, all terms $O\left(\Delta^{4}\right)$ must be considered.

It is interesting to note that two quantities here are in the ratio of $4: 1$. These are the weighted overall splittings $\Delta E$ of the energy levels:

$$
\left[(\Delta E)_{C_{4 v}}-(\Delta E)_{C_{3 v}} V\left[(\Delta E)_{C_{2 v}}-(\Delta E)_{C_{3 v}}\right]=4 / 1 .\right.
$$

Also, and perhaps of more significance, the ratio for the energies of the highest crystal field components is

$$
\left[E\left(C_{4 v}\right)-E\left(C_{3 v}\right)\right] /\left[E\left(C_{2 v}\right)-E\left(C_{3 v}\right)\right]=4 / 1 .
$$

B. Combined splitting of $p$ orbitals and mixing with $s$ orbitals

1. $C_{4 v}$

Let $U$ denote the energy difference $E_{p}-E_{s}$, then

$$
\begin{aligned}
& \langle s, 0|V| s, 0\rangle=0, \\
& \langle s, 0|V| p, 0\rangle=4 a \sin \Delta / \sqrt{3} .
\end{aligned}
$$

The energy matrix is

$$
\left.\begin{array}{cc}
(4 a / 3) \sin \Delta & 0 \\
0 & 0 \\
U+(12 b / 5) \sin ^{2} \Delta & 0 \\
0 & U-(6 b / 5) \sin ^{2} \Delta
\end{array}\right]
$$

The energy matrix is

$\left.\begin{array}{cc}2 \sqrt{2} a \sin \delta & 0 \\ 0 & 0 \\ U+(6 b / 5) \sin ^{2} \delta & 0 \\ 0 & U-3 b \sin ^{2} \delta\end{array}\right]$,




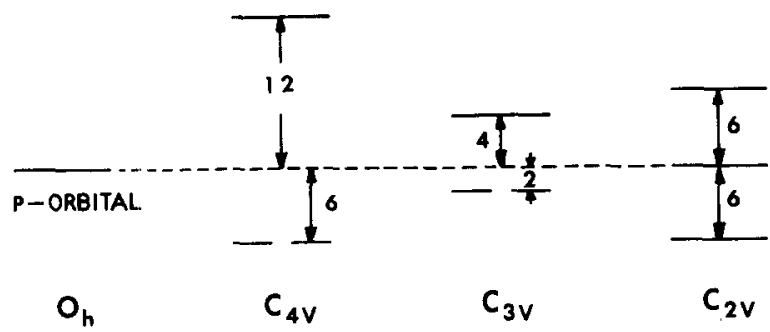

FIG. 9. Splitting of energy levels of a single $p$ orbital at various symmetries due to $l=2$ part of crystal field. The energy units are $\sin ^{2} \Delta / 5$ [Eq. (38)], with ligand having $R_{n}=1$ and $Q_{n}=-1[\mathrm{Eq}$. (35)]. The upper level is the component which mixes with an $s$ orbital by the $l=1$ part of the field.

where the threefold axis is the quantitation axis. Therefore to second order the ground state energy is

$$
E\left(C_{3 v}\right)=-\left(8 a^{2} \sin ^{2} \delta / U\right)\left\{1-(6 b / 5 U) \sin ^{2} \delta\right\} .
$$

Using Eq. (38) and keeping only terms up to $0\left(\Delta^{4}\right)$,

$E\left(C_{3 v}\right)=-\frac{16 a^{2} \sin ^{2} \Delta}{3 U}\left[1-\frac{4 b \sin ^{2} \Delta}{5 U}\right]-\frac{16 a^{2} \Delta^{3} \sin \Delta}{27 U}$.

\section{3. $C_{2 v}$}

An analysis similar to that for $C_{3 v}$ and $C_{4 v}$ can be made using the appropriate matrix elements in Eq. (34), yielding a second-order ground state energy

$E\left(C_{2 v}\right)=-8 a^{2} / U\left(\sin \alpha+2^{-1 / 2} \sin \beta\right)^{2}\left\{1-(6 b / 5 U) \sin ^{-2} \beta\right\}$,

which after use of Eq. (39) and keeping only terms up to $O\left(\Delta^{4}\right)$ yields

$E\left(C_{2 v}\right)=-\frac{16 a^{2} \sin ^{2} \Delta}{3 U}\left[1-\frac{6 b \sin ^{2} \Delta}{5 U}\right]-\frac{4 a^{2} \Delta^{3} \sin \Delta}{9 U}$.

The results for all three symmetries are summarized in Table V. Note that again the relationship of Eq. (29) is satisfied.

For small radial displacements, such that $\sin \Delta=\Delta$, the angular barrier $E\left(C_{4 v}\right)-E\left(C_{3 v}\right)$ is $8 b \Delta^{2} / 5 U+\Delta^{2} / 9$ in units of $16 a^{2} \Delta^{2} / 3 U$ (Table V), with $\Delta$ in radians. Since $8 b \Delta^{2} / 5$ is the shift in the first order energy of the upper $p$ component in going from $C_{3 v}$ to $C_{4 v}$ [Eqs. (42) and (44)], the first term dominates if this shift is more than 9 times the product of $\Delta^{2}$ and the unperturbed $s-p$ interval. For our parameter choices and for angles up to $15^{\circ}$, numerical evaluation shows that the first term indeed dominates.

In summary, there are two contributions to the angular variation in the second-order energy of the mostly

TABLE V. Ground state energies at different symmetries for $(s, p)$ system (in units of $16 a^{2} \sin ^{2} \Delta / 3 U$ ).

\begin{tabular}{lll}
\hline Symmetry & Ground state energy & $E_{0}-E_{0}\left(C_{3 v}\right)$ \\
\hline$C_{4 v}$ & $-1+12 b \sin ^{2} \Delta / 5 U$ & $8 b \sin ^{2} \Delta / 5 U+\Delta^{3} / 9 \sin \Delta$ \\
$C_{2 v}$ & $-1+6 b \sin ^{2} \Delta / 5 U-\Delta^{3} / 12 \sin \Delta$ & $2 b \sin ^{2} \Delta / 5 U+\Delta^{3} / 36 \sin \Delta$ \\
$C_{3 v}$ & $-1+4 b \sin ^{2} \Delta / 5 U-\Delta^{3} / 9 \sin \Delta$ & 0 \\
\hline \hline
\end{tabular}

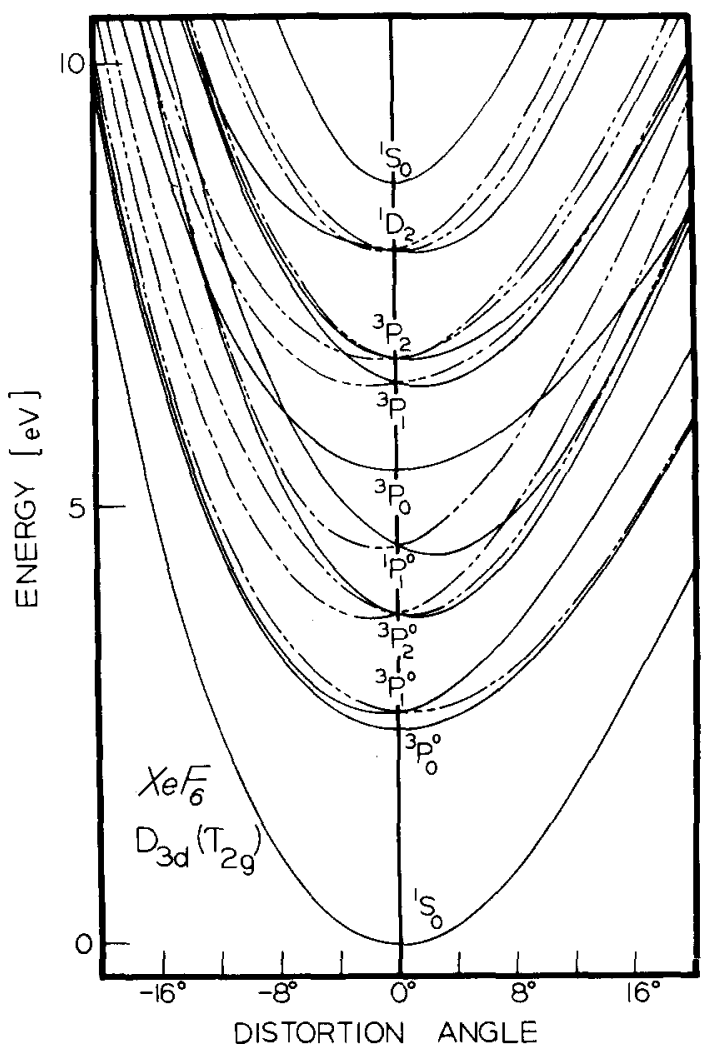

FIG. 10. Electronic energy in electron volt vs the $t_{2 g}$ bending angle $\delta$, producing symmetry $D_{3 d}$ except at the $O_{h}$ origin. Energy parameters are the same as those in Fig. 5. Calculations were made at $\delta=0, \pm 2^{\circ}, \pm 4^{\circ}, \pm 8^{\circ}, \pm 12^{\circ}, \pm 16^{\circ}$, and $\pm 20^{\circ}$, with positive $\delta$ for prolate structures and negative $\delta$ for oblate structures. The solid lines denote nondegenerate states, with dashed lines for doubly degenerate states. For this structure the ligand repulsion curve virtually coincides with the groundstate curve.

$s$ orbital. First, the $s-p$ mixing matrix elements, associated with the $l=1$ part of the crystal field, are dependent to a small degree on the direction in $t_{1 u}$ bending space, that is, on the angular variables of Eq. (28). This effect is small and becomes zero when Eq. (35) is used as an approximation to Eq. (36). Second, the energy denominators in the second-order energy expression vary with the direction in $t_{1 u}$ bending space due to a variation in the $l=2$ part of the crystal field. Thus the first-order splitting of the $p$ orbital modulates the second-order energy of the $s$ orbital. This effect does not vanish with the approximation implied by Eq. (35) and dominates for our parameter choices. The $l=1$ part of the field mixes the $s$ orbital only with the highest energy $p$ orbital for each structure in Fig. 9. Thus the energy denominator is greatest for $C_{3 v}$, resulting in a lower energy for the mostly $s$ orbital. The fraction of $p$ character in this orbital is also greater for $C_{3 v}$, meaning that the very open face of a $C_{3 v}$ structure leads to a greater "sticking out" of the electron density.

\section{POTENTIAL ENERGY CURVES FOR $T_{2 g}$ AND $T_{2 u}$ BENDING}

We set the conditions for the $t_{2 \xi}$ mode of bending such that: (1) $r_{\mathrm{X}-F}=1.89 \times 10^{-8} \mathrm{~cm}$, and (2) $\alpha_{15}=\alpha_{24}=\ldots=\Delta$. 


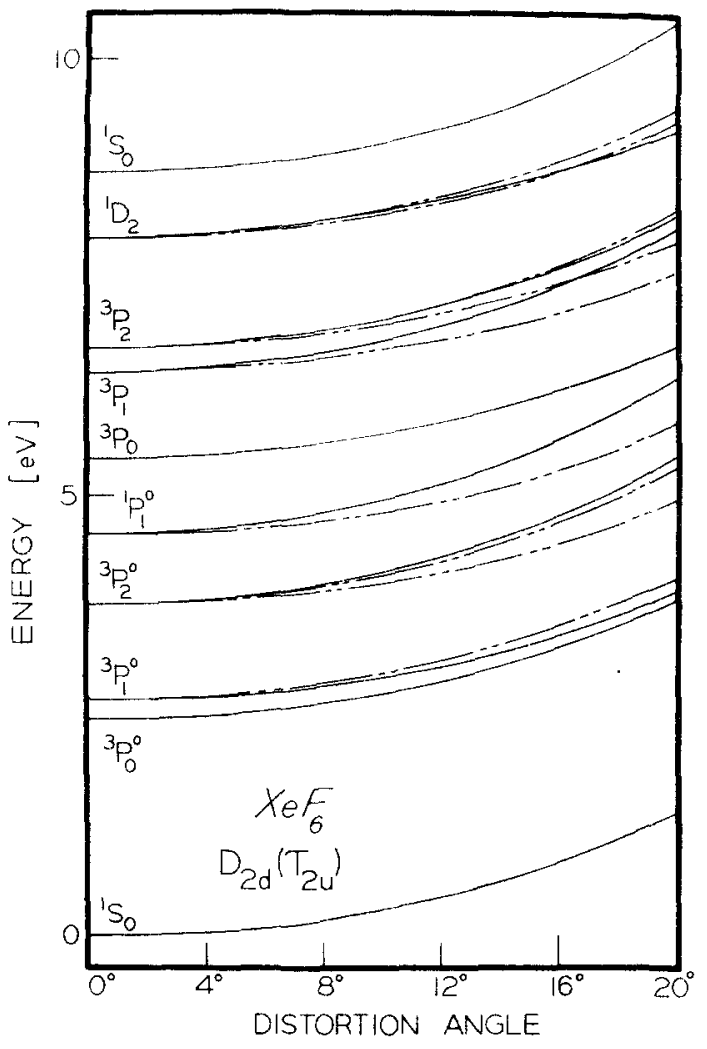

FIG. 11. Curves as in Fig. 10, but vs the $t_{2 u}$ bending angle $\Delta$. Parameters are as in Fig. 4. As for $t_{2 g}$ bending the groundstate curve coincides with the ligand repulsion curve.

We used the assumed best set of parameters $(\Delta E=3.5$ $\left.\mathrm{eV}, F_{2}=0.5 \mathrm{eV}, F_{1}=0.08 \mathrm{eV}, \zeta=0.87 \mathrm{eV}\right)$. The molecular symmetry in this case is $D_{2 h}$, with the results showing the expected Jahn-Teller splitting of the excited degenerate states. The ground state has its energy minimum at $O_{k}$. These curves are not shown, as we plot instead the energies for $D_{3 d}\left(t_{2 g, x}+t_{2 \xi, y}+t_{2 g, z}\right)$ structures using the same set of parameters (Fig. 10). The results show that the lowest triplet state ${ }^{3} T_{1 u}$ is approximately $2.5 \mathrm{eV}$ or $20000 \mathrm{~cm}^{-1}$ above the ground state ${ }^{1} A_{1 g}$ at $O_{h}$; as the molecule is distorted away from $O_{h}$ along the $D_{3 d}$ axis only about $\pm 10^{\circ}$ (the prolate $D_{3 d}$ and the oblate $D_{3 d}$ ), the lowest triplet component is still about $2.2 \mathrm{eV}$ or $17000 \mathrm{~cm}^{-1}$ above the ground state. There is no low-lying triplet state to be found that is either thermally accessible to the ground state or crossing the ground state within even a large range of angular distortion. The force constant estimated from the ground state energy curve is about $3.0 \mathrm{mdyn} / \AA$. Thus yields a frequency of $\nu_{5}=259 \mathrm{~cm}^{-1}$. We note al so that in this case the ${ }^{1} A_{1 g}$ ground state energy curve is the same as that of the ligand repulsion $V_{\text {rep }}$. We also remember that the experimental result of $\nu_{5}$ for $\mathrm{TeCl}_{6}^{-2}$ is $131 \mathrm{~cm}^{-1}$ (force constants: $F=0.13 \mathrm{mdyn} / \AA, K$ $=1.18 \mathrm{mdyn} / \AA, H=0.0 .02 \mathrm{mdyn} / \AA$, ); and that for $\mathrm{TeBr}_{6}^{-2}$ is $70 \mathrm{~cm}^{-1}$ (force constants: $F=0.09 \mathrm{mdyn} / \AA$, $K=0.97 \mathrm{mdyn} / \AA, H=0.02 \mathrm{mdyn} / \AA$. ). ${ }^{59}$

We set the conditions for the $t_{2 u}$ mode of bending such that: (1) $r_{\mathrm{X}-\mathrm{F}}=1.89 \times 10^{-8} \mathrm{~cm}$, and (2) $\alpha_{13}=\alpha_{12}=\cdots$ $=\Delta$. In Fig. 11, we plot the electronic energy results for the $t_{2 u}$ mode for the same set of parameters used for the $t_{2 g}$ mode. The molecular symmetry in this case is $D_{2 d}$, with no $s-p$ mixing even though the molecule lacks an inversion center. It is noticed that all the energy curves of this mode are rather flat with their minima at $O_{h} \mathrm{im}-$ plying small force constants and fluctuation of geometry around $O_{h}$. Also it is to be noted that in this case the ground state ${ }^{1} A_{1 g}$ energy curve is the same as that of the ligand repulsion potential. For this set of parameters the ground state force constant is estimated to be about $0.90 \mathrm{mdyn} / \AA$, thus gives a frequency $\nu_{6}=140 \mathrm{~cm}^{-1}$. It is interesting to note that the frequency ratio of $\nu_{5}$ to $\nu_{6}$ is 1.8 . This is a ratio that the other regular hexafluorides also share. ${ }^{6,58}$

\section{COUPLING OF $T_{1 u}$ AND $T_{2 g}$ BENDING MODES}

As discussed by Bartell and Gavin, ${ }^{17}$ cubic and quartic terms are of particular importance in the potential energy function for $X e F_{6}$. Although quartic and higher terms in a single vibrational coordinate are implicitly considered in our treatments of the $t_{1 u}, t_{2 g}$, and $t_{2 u}$ bending spaces, we have so far neglected terms which involve the coupling of these coordinates. First consider the coupling of the $t_{1 u}$ and $t_{2 g}$ bending modes. Bartell and Gavin represent the interaction by the terms

$$
V_{445}=k_{445}\left(Q_{4 x} Q_{4 y} Q_{5 z}+Q_{4 x} Q_{4 \varepsilon} Q_{5 y}+Q_{4 y} Q_{4 \varepsilon} Q_{5 x}\right)
$$

and

$$
\begin{aligned}
V_{4455}=k_{4455}\left[\left(Q_{4 x}^{2}+Q_{4 y}^{2}\right) Q_{5 z}^{2}+\left(Q_{4 x}^{2}\right.\right. & \left.+Q_{4 z}^{2}\right) Q_{5 y}^{2} \\
& \left.+\left(Q_{4 y}^{2}+Q_{4 z}^{2}\right) Q_{5 x}^{2}\right],
\end{aligned}
$$

where the subscript 4 and 5 denote $t_{1 u}$ and $t_{2 \xi}$ bending coordinates, respectively, while the subscripts $x, y$, and $z$ denote Cartesian components. For $t_{2 q}$ the symbols $x, y, z$ denote $y z, x z$, and $x y$, respectively. The above terms must be added to the terms appropriate to the pure $t_{1 u}$ and $t_{2 \varepsilon}$ spaces. For a $C_{4 v}$ structure, say with $z$ as the fourfold axis, $Q_{4 x}=Q_{4 y}=0$, with all components of $Q_{5}=0$. Thus

$$
V_{445}\left(C_{4 v}\right)=V_{4455}\left(C_{4 v}\right)=0 \text {. }
$$

For a $C_{2 v}$ structure, say with $x+y=(110)$ as the twofold axis, $Q_{4 x}=Q_{4 y}, Q_{4 z}=Q_{5 x}=Q_{5 y}=0$. Thus

$$
\begin{aligned}
& V_{445}\left(C_{2 v}\right)=\frac{1}{2} k_{445}\left(Q_{4}\right)_{110}^{2}\left(Q_{5}\right)_{100} \\
& V_{4455}\left(C_{2 v}\right)=k_{4455}\left(Q_{4}\right)_{110}^{2}\left(Q_{5}\right)_{110}^{2} .
\end{aligned}
$$

For a $C_{3 v}$ structure, say with $x+y+z=(111)$ as the threefold axis, $Q_{4 x}=Q_{4 y}=Q_{4 x}$ and $Q_{5 x}=Q_{5 y}=Q_{5 z}$. Thus

$$
\begin{aligned}
& V_{445}\left(C_{3 v}\right)=k_{445}\left(Q_{4}\right)_{111}^{2}\left(Q_{5}\right)_{111} \\
& V_{4455}\left(C_{3 v}\right)=2 k_{4455}\left(Q_{4}\right)_{111}^{2}\left(Q_{5}\right)_{111}^{2} .
\end{aligned}
$$

In Eqs. (59) and (60), the subscripts (110) and (111) denote the appropriate directions for the bending coordinates; the least obvious might be $\left(Q_{5}\right)_{110}$, which is the same as $Q_{5 g}$. The expressions for $C_{3 v}$ are similar to those for $C_{2 v}$, but the former are larger by a factor of 2.

We now consider in detail the ground state energy of $\mathrm{XeF}_{6}$ in the 2-space defined by the vibrational coordinates $\left(Q_{4}\right)_{111}$ and $\left(Q_{5}\right)_{111}$, which are shown in Fig. 12. The combination of these coordinates permits one triad 

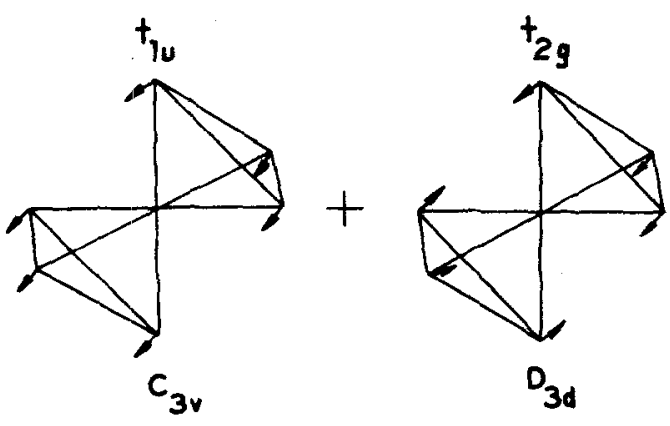

FIG. 12. An example of coupled bending modes: the $t_{1 u}\left(C_{3 v}\right)$ with $t_{2 g}\left(D_{3 d}\right)$ to produce a $C_{3 v}$ structure with one very open face.

of ligands to "open up" widely without forcing the other triad to "close up" to the degree required by pure $t_{1 u}$ bending. The ground state energies calculated using the same parameters as in Figs. 4, 10, and 11 are shown as contours in Fig. 13. There is a right-left symmetry but not a top-bottom symmetry, as changing the sign of the $t_{2 g}$ coordinate changes the shape of the molecule. Points along the vertical axis thus correspond to those in Fig. 10. We note that whereas the pure $t_{1 u}$ minimum occurs on the horizontal axis at an angle of $8.9^{\circ}$, with an energy $0.23 \mathrm{eV}=1855 \mathrm{~cm}^{-1}$ below that for $O_{h}$ symmetry, the minimum in the 2-space occurs at a $t_{1 u}$ angle of $13.6^{\circ}$ and a $t_{2 g}$ angle of $5.6^{\circ}$ with an energy of $0.46 \mathrm{eV}=3710 \mathrm{~cm}^{-1}$ below that for $O_{h} \mathrm{sym-}$ metry. Thus one triad of ligands has opened up from a polar angle of $63.6^{\circ}$ for pure $t_{1 u}$ bending to an angle of $73.9^{\circ}$ (for $O_{h}$ the angle is $54.73^{\circ}$ ), while the other triad, at a polar angle of $134.2^{\circ}$ for pure $t_{1 u}$, remains almost fixed at $133.3^{\circ}$. In the process, the energy dif ference between $C_{4 v}$ and $C_{3 v}$ structures has increased from about $160 \mathrm{~cm}^{-1}$ in pure $t_{1 u}$ space to about $2015 \mathrm{~cm}^{-1}$.
Thus a barrier results which is too high to be thermally surmounted at room temperature. However, as Fig. 7 shows, the route from one $C_{3 v}$ minimum to another need not pass through $C_{4 v}$.

It is difficult to reach strong conclusions here, as cubic and quartic terms coupling the $t_{1 u}$ bending mode to modes other than $t_{2 g}$ can modify these results. Specifically, cubic and quartic coupling between the $t_{1 u}$ bending mode and the $e_{g}$ stretching mode stabilizes $C_{2 v}$ and $C_{4 v}$ structures, but not $C_{3 v}$, while quartic coupling of the $t_{1 u}$ and $t_{2 u}$ bending modes is important for $\mathrm{C}_{2 v^{*}}$. Thus without performing extensive further calculations little more can be said about the barriers to pseudorotation.

\section{SUMMARY}

The results of these simple crystal field calculations support the view that the $\mathrm{XeF}_{6}$ molecule has a nonoctahedral equilibrium structure, with distortions mostly along its $t_{1 u}$ bending coordinates. It is estimated that for this vibration there is a negative force constant at the $O_{h}$ symmetry of about $-1.0 \mathrm{mdyn} / \AA$ and an equilibrium structure corresponding to a nearly $9^{\circ}$ deformation away from $O_{h}$ symmetry, producing a minimum potential trough approximately $1860 \mathrm{~cm}^{-1}$ deep for a $C_{3 v}$ structure and with curvature of $1.8 \mathrm{mdyn} / \AA$. Using a one-dimensional approximation to the potential curve, this trough has a radial vibrational zero-point energy of about $51 \mathrm{~cm}^{-1}$ and an energy spacing for the lower vibrational levels of about $109 \mathrm{~cm}^{-1}$. It should be noted that this minimum energy trough is not isotropic, since there is an energy difference of about $160 \mathrm{~cm}^{-1}$ between $C_{4 v}$ and $C_{3 v}$ structures, and a difference of about $40 \mathrm{~cm}^{-1}$ between $C_{2 v}$ and $C_{3 v}$ structures. The $C_{4 v}, C_{2 v}$, and $C_{3 v}$ structures correspond to a maximum, a saddle point and a minimum, respectively, in the space of the two angu-

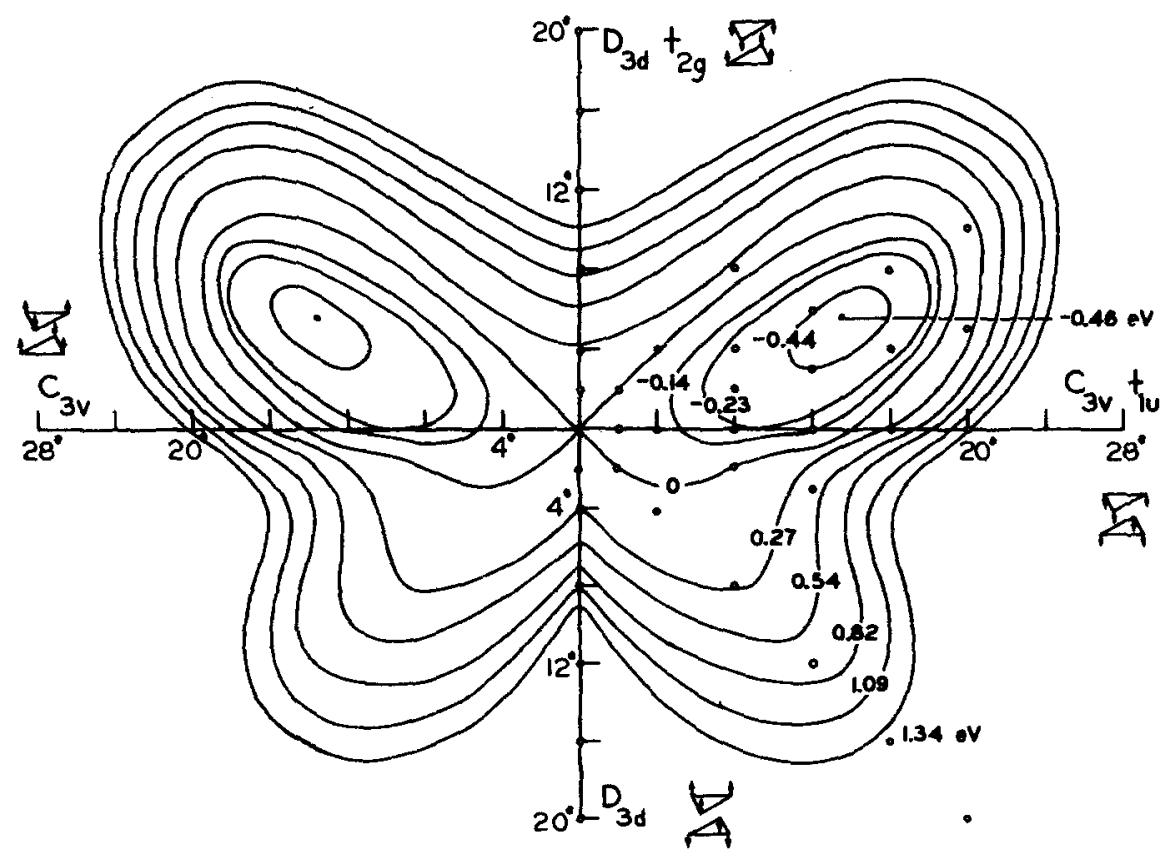

FIG. 13. Potential energy surface in the two-dimensional space of the $t_{1 u}(111)$ and $t_{2 g}$ (111) bending coordinates. Small open circles indicate geometries for which calculations were made, after which approximate contours were sketched. 
lar components of the $t_{1 u}$ bending vibration. These energy differences in pure $t_{1 u}$ bending space correspond to barriers which are in the range of thermal energies. If the barriers are this low, the molecule can move with relative ease in this energy space, passing through six $C_{4 v}$, eight $C_{3 v}$, and twelve $C_{2 v}$ structures. This is equivalent to saying that the lone pair is rapidly going through the faces and edges of an octahedron, or that the molecule is rapidly changing and inverting its structures along the path $C_{3 v} \rightarrow C_{2 v} \rightarrow C_{3 v} \rightarrow C_{2 v} \rightarrow \cdots$ of minimum hindrance. Thus, the average electric dipole moment for the molecule should be zero. However, the $C_{3 v}$ structure is further stabilized by coupling between the $t_{1 u}$ and $t_{2 g}$ bending modes to an extent that would preclude pseudorotation at room temperature. Further studies need to be made to estimate the stabilization of the $C_{2 v}$ and $C_{4 v}$ structures by similar couplings to other modes, such as the $t_{2 u}$ bending mode and the $e_{g}$ stretching mode. The pure $t_{2 g}$ and $t_{2 u}$ force constants are estimated to be 3.0 and $0.9 \mathrm{mdyn} / \AA$, corresponding to vibrational frequencies of 259 and $140 \mathrm{~cm}^{-1}$ for $t_{2 g}$ and $t_{2 u}$, respectively.

The results of this model fail to locate a low-lying triplet component which is thermally accessible to the ground state, but instead indicate that the deformation of $\mathrm{XeF} \mathrm{F}_{\mathrm{B}}$ from $O_{h}$ symmetry is largely through $t_{1 u}$ bending, which is accompanied by a mixing of xenon $5 s$ and $5 p$ orbitals.

Results for other $\mathrm{Xe}(+6)$ systems, including $\mathrm{XeF}_{8}^{-2}$, $\mathrm{XeOF}_{4}, \mathrm{XeO}_{2} \mathrm{~F}_{2}$, and $\mathrm{XeO}_{3}$, are considered in the second paper in this series. Finally, the important topic of the intensities and band shapes for the electronic absorption transitions, together with their temperature dependencies, is the subject of the third paper.

\section{ACKNOWLEDGMENTS}

The authors wish to thank Dr. Harold Basch for supplying them with xenon atom SCF wavefunctions and with several computer programs for evaluating integrals using Gaussian basis sets. Particular gratitude is due to Professor Lawrence Bartell for his numerous suggestions and helpful comments.

${ }^{1}$ H. H. Hyman, (Ed.), Noble-Gas Compounds (University of Chicago Press, Chicago, 1963).

${ }^{2}$ J. G. Malm, H. Selig, J. Jortner, and S. A. Rice, Chem. Rev. 65, 199 (1965).

${ }^{3}$ H. H. Claassen, The Noble Gases (Heath, Lexington, MA., 1966).

${ }^{4}$ H. H. Hyman, Physical Chemistry: An Advanced Treatise, Vol. V, edited by H. Eyring, D. Henderson, and W. Jost (Academic, New York, 1970), pp. 589-662.

${ }^{5}$ C. A. Coulson, J. Chem. Soc. 1964, 1442.

${ }^{6} \mathrm{~B}$. Weinstock and G. L. Goodman, Adv. Chem. Phys. 9, 169 (1965).

${ }^{7}$ F. Schreiner, D. W. Osborne, J. G. Malm, and G. N. Mc-
Donald, J. Chem. Phys. 51, 4838 (1969).

${ }^{8}$ H. H. Hyman and L. A. Quarterman, Ref, 1, pp. 275-278.

${ }^{9}$ D. F. Smith, Ref. 1, pp. 39-46.

${ }^{10}$ H. H. Claassen, Ref. 1, pp. 304-305.

${ }^{11}$ H. Kim, H. H. Claassen, and E. Pearson, Inorg. Chem. 7, 616 (1968).

${ }^{12}$ B. Weinstock, E. E. Weaver, and C. P. Knop, Inorg. Chem. 5, 2189 (1966).

${ }^{13}$ E. L. Gasner and H. H. Claassen, Inorg. Chem. 6, 1937 (1967).

${ }^{14}$ H. H. Claassen, G. L. Goodman, and H. Kim, J. Chem. Phys, 56, 5042 (1972).

${ }^{15}$ L. S. Bartell, R. M. Gavin, Jr., H. H. Thompson, and C. L. Chernick, J. Chem. Phys. 43, 2547 (1965).

${ }^{16}$ R. M. Gavin, Jr. and L. S. Bartell, J. Chem. Phys. 48, 2460 (1968).

${ }^{17}$ L. S. Bartell and R. M. Gavin, Jr., J. Chem. Phys. 48, 2466 (1968).

${ }^{18}$ K. Hedberg, S. H. Peterson, R. R. Ryan, and B. Weinstock, J. Chem. Phys. 44, 1726 (1966).

${ }^{19}$ G. Engle, Z. Krist. 90, 341 (1935).

${ }^{20}$ E. E. Aynsley and A. C. Hazell, Chem. and Ind. (Lond.) 1963, 611 .

${ }^{21}$ I. D. Brown, Can. J. Chem. 42, 2758 (1964).

${ }^{22} \mathrm{~S}$. Lawton and R. Jacobson, Inorg. Chem. 5, 743 (1966).

${ }^{23}$ G. L. Goodman, J. Chem. Phys. 56, 5038 (1972).

${ }^{24}$ R. F. Code, W. E. Falconer, W. Klemperer, and I. Ozier, J. Chem. Phys. 47, 4955 (1967).

${ }^{25}$ W. E. Falconer, A. Btichler, J. L. Stauffer, and W. Klemperer, J. Chem. Phys. 48, 312 (1968).

${ }^{26}$ C. R. Brundle, G. R. Jones, and H. Basch, J. Chem. Phys. 55, 1098 (1971).

${ }^{27}$ J. Berkowitz, W. A. Chupka, P. M. Guyon, J. H. Holloway, and R. Spohr, J. Phys. Chem. 75, 1461 (1971).

${ }^{28}$ G. M. Begun and R. N. Compton, J. Chem. Phys. 51, 2367 (1969).

${ }^{29}$ R. J. Gillespie, Ref. 1, pp. 333-339.

${ }^{30}$ L. S. Bartell, J. Chem. Phys. 46, 4530 (1967).

${ }^{31}$ L. S. Bartell, J. Chem. Educ. 45, 754 (1968).

${ }^{32}$ E. U. Condon and G. H. Shortley, The Theory of Atomic Spectra, (Cambridge U. P., New York, 1951).

${ }^{33} \mathrm{~J}$. C. Slater, Quantum Theory of Atomic Structure, Vol. 1, (McGraw-Hill, New York, 1960).

${ }^{34}$ L. L. Lohr, Jr., Coord. Chem. Rev. 8, 241 (1972).

${ }^{35}$ M. Blume and R. E. Watson, Proc. R. Soc. Lond. A270, 127 (1962), A271, 565 (1963).

${ }^{36}$ The results of this section draw heavily upon J. S. Griffith, The Theory of Transition Metal Ions (Cambridge U, P., New York, 1961).

${ }^{37}$ C. E. Moore, Natl. Bur. Stand. Circ. No. 467, 3, 55-123 (1958).

${ }^{38} \mathrm{C}$. K. Jorgensen, Modern Aspects of Ligand Field Theory (North Holland, Amsterdam, 1971), pp. 293-313.

${ }^{39} \mathrm{C}$. K. Jorgensen, Absorption Spectra and Chemical Bonding in Complexes (Pergamon, Elmsford, NY, 1962), pp. 185-189.

${ }^{40} \mathrm{~J}$. Owen, Proc. R. Soc. (Lond.) 227, 183 (1954).

${ }^{41}$ T. M. Dunn, J. Chem. Soc. 1959, 623 .

${ }^{42}$ D. P. Craig and E. A. Maguson, Discuss. Faraday Soc. 26, 116 (1958); and J. Chem. Soc. 1956, 4895.

${ }^{43}$ R. A. Walton, R. W. Mathes, and C. K. Jørgensen, Inorg. Chim. Acta 1, 355 (1967).

${ }^{44}$ Computed by us from xenon neutral atom Gaussion basis set SCF wave functions supplied by $\mathrm{H}$. Basch,

${ }^{45}$ E. A. Boudreaux, Ref. 1, pp. 354-357.

${ }^{46}$ C. L. Chernick, J. G. MaIm, and I. Sheft, J. Am. Chem. Soc. 85, 110 (1963).

${ }^{47}$ R. G. Pearson, J. Am. Chem. Soc. 91, 4947 (1969).

${ }^{48}$ H. Basch, J. W. Moskowitz, C. Hollister, and D. Hankin, J. Chem. Phys. 55, 1922 (1971).

${ }^{49}$ D. A. Couch, C. J. Wilkins, and H. Gray, J. Am. Chem. Soc. 92, 307 (1970).

${ }^{50} \mathrm{~J}$. W. Hastie, R. H. Hague, and J. L. Margrave, J. Mol. 
Spectrosc. 29, 152 (1969).

${ }^{51}$ R. Zollweg and L. S. Frost, J. Chem. Phys. 50, 3280 (1969). ${ }^{52}$ Supplied by H. Basch, and similar to the basis functions used in Ref. 48 .

${ }^{53}$ In the electron diffraction results of Ref. 17 , it is pointed out that $t_{i_{u}}$ stretching is less important that $t_{1 u}$ bending and $e_{g}$ stretching is less important than $t_{2 g}$ bending.

${ }^{54}$ U. Öpik and M. H. L. Pryce, Proc. R. Soc. Lond. A238,
425 (1957).

${ }^{55}$ R. F. W. Bader, Mol. Phys. 3, 137 (1960).

${ }^{56}$ R. F. W. Bader, Can. J. Chem. 40, 1164 (1962).

${ }^{57}$ R. G. Pearson, J. Am. Chem. Soc. 91, 1252 (1969).

${ }^{58}$ H. H. Claassen, G. L. Goodman, J. H. Holloway, and H. Selig, J. Chem. Phys. 53, 341 (1970).

${ }^{59}$ P. J. Hendra and Z. Jovic, J. Chem. Phys. 1968 (A) 600 (1968). 